Scientific Report No. 104

\title{
Homogenization Analysis of Electromagnetic Strip Gratings
}

\author{
by \\ Ronald R. DeLyser and Edward F. Kuester \\ Electromagnetics Laboratory \\ Department of Electrical and Computer Engineering \\ Campus Box 425 \\ University of Colorado \\ Boulder, Colorado 80309-0425
}

May 1990 


\begin{abstract}
The boundary conditions for the average fields at a periodic metal grating of ribbon conductors are developed. The method of homogenization is applied: the technique of multiple scales is used to expand the fields scattered from the periodic structure in a power series. This process leads to separation of the fine structured boundary layer fields from the average fields which exist at a distance from the structure. Solution of these separate problems leads to the boundary conditions for the average fields which are referred to in the literature as "equivalent" or "averaged" boundary conditions.
\end{abstract}

\title{
1 INTRODUCTION
}

This paper addresses the development of boundary conditions of the average or non-boundary layer fields of a periodic metal grating of strips using the method of homogenization. We will show that the fields at a distance from the grating (which are also the "average" fields of the structure) can be described in terms of a homogeneous structure exhibiting a special "equivalent boundary condition." The only assumption for the derivation is that the structure be periodic with respect to one or more of the dimensions and with period small when compared to a wavelength. This assumption is often true in microwave applications of grids or gratings but not usually at optical wavelengths.

The problem of analyzing gratings has a long history of which we will limit this introduction to works with particular relevance to the topic of this paper. The papers cited fall into three convenient categories: (1) investigation of the high-frequency diffracted fields, (2) equivalent network formulations, and (3) equivalent boundary conditions formulations. Of papers of the first type, there are many contributors in the field of optics who will not be cited here.

Lamb, in his classic paper of 1898 [1], used conformal mapping techniques to determine the static fields of a ribbon grating. His drawings of the field lines for the ribbons parallel and perpendicular to the plane of incidence are quite illuminating. He also investigated the problem of different media on either side of the grid. Primich [2] investigated transmission and reflection properties using a theory based on a variational method, and compared 
this theory to experiment with good results. Others, [3], [4], and [5], used numerical techniques to compute the diffracted fields.

Oliner has been particularly active in analyzing gratings using a multimode network formulation. A series of papers [6] - [9] authored by Oliner and/or his colleagues has offered quite a different point of view on this topic with the added benefit of novel solutions to certain integral equations. Their results compare favorably with independent numerical results.

The papers which formulate equivalent boundary conditions are quite numerous. Sakurai [10] follows the lead of Lamb but takes the theory one step further in developing the equivalent boundary conditions for the ribbon conductor in free space. Sivov [11], Kontorovich [12], and Wainstein [13] addressed gratings of more general cross sections, also only in free space. Adonina and Shcherbak [14], [15] have developed their theory for different materials on either side of a plane metallic grating by reducing the problem to "two inhomogeneous problems of conjugation." Astrakhan [16] derived expressions, using averaged boundary conditions, for the reflection coefficients of plane wire grids of rectangular and square cells as well as parallel wires. Experiments compared well with the theory.

The method of homogenization as applied to electromagnetic problems is relatively new. Results applicable to electromagnetics problems are published in [18] for periodic structures, [19] for a corrugated impedance surface and in [20] to the static problem for a wire grid. We apply it here to a two dimensional periodic structure of ribbon conductors.

\section{THE GEOMETRY AND EXPANSION OF THE SCATTERED FIELDS}

\section{$2.1 \quad$ Introduction}

Homogenization is based on using the technique of multiple scales to solve problems in media which include periodic structures. (For detailed mathmatical discussions see [21] and [22].) In this method, the scattered fields are expanded as functions of the period of the structure. In this way, one can separate the the macroscopic characteristics of the average field from the periodic aspects arrising from the non-homogeneous structure. Thus, in examining these characteristics, we can derive boundary conditions for the 


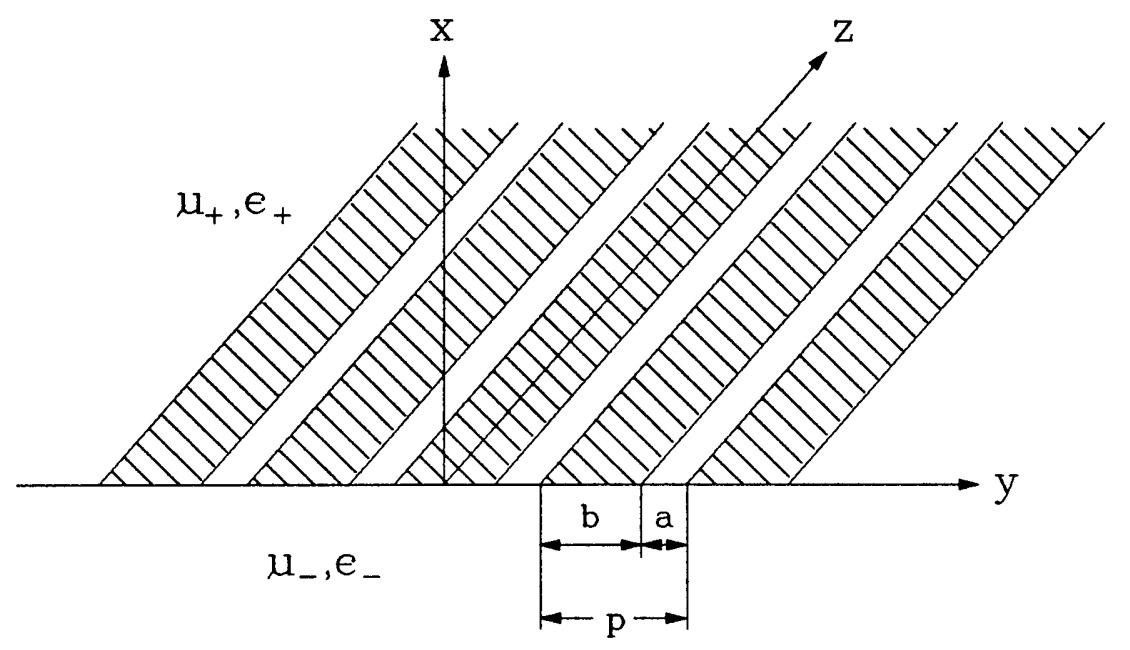

Figure 1: A Periodic Grid of Ribbon Conductors.

average fields which are refered to as "equivalent boundary conditions" for the structure as if it were homogeneous.

For the problem at hand, a grid of period $p$ consisting of infinitely long conductors of zero thickness, width $b$, and spacing $a$ is placed between two different media as shown in Figure 1. The different media have material properties which will be noted as having a + subscript for $x>0$ and a subscript for $x<0$. Likewise, the fields in these media will also be noted with these subscripts. ${ }^{1}$ Incident electric and magnetic fields in the region where $x>0$ are postulated. They in turn cause a scattered field everywhere. The incident and scattered fields are linearly combined to give the total fields which can be written

$$
\left.\begin{array}{rl}
\bar{E}_{+}^{t}=\bar{E}_{+}^{i}+\bar{E}_{+}^{s} & x>0 \\
\bar{E}_{-}^{t}=\bar{E}^{s} & x<0 \\
\bar{H}_{+}^{t}=\bar{H}_{+}^{i}+\bar{H}_{+}^{s} & x>0 \\
\bar{H}_{-}^{t}=\bar{H}_{-}^{s} & x<0 .
\end{array}\right\}
$$

\footnotetext{
${ }^{1}$ In the derivations to follow, unless special emphasis is needed, the equations will be written without subscripts. The corresponding equations for the + and - media will be assumed.
} 


\subsection{Expansion of the scattered fields}

We make the stipulation that the period of the grating, $p$, is small compared to the radiation wavelength, $\lambda$. By virtue of this condition, we postulate that for a given incident plane wave there will be, at a distance far removed from the grating, a reflected and transmitted wave only, with no apparent diffracted fields. That is to say, the fine structure of the fields is confined to a boundary layer close to the grid. Therefore, we assume that the scattered fields are functions of a "slow" variable and a "fast" variable. The fast variable is defined as

$$
\tilde{r}=\frac{r}{p} \equiv \tilde{x} \text { or } \tilde{y} \text { or } \tilde{z} \text {, and } r \equiv x \text { or } y \text { or } z
$$

where $x, y$, and $z$ are the usual cartesian coordinates. Even though the fast variable depends on the slow variable, we will temporarily assume that they are independent. This is probably the most critical assumption in the homogenization theory and indeed is the basis for the separation of the average fields from the periodic, fine structured, fields. The del vector operator is then given as

$$
\nabla=\nabla_{r}+\frac{1}{p} \nabla_{\tilde{r}}
$$

where

$$
\left.\begin{array}{c}
\nabla_{r} \equiv \frac{\partial}{\partial x} \hat{a}_{x}+\frac{\partial}{\partial y} \hat{a}_{y}+\frac{\partial}{\partial z} \hat{a}_{z} \\
\nabla_{\tilde{r}} \equiv \frac{\partial}{\partial \tilde{x}} \hat{a}_{\tilde{x}}+\frac{\partial}{\partial \tilde{y}} \hat{a}_{\tilde{y}}
\end{array}\right\}
$$

because there is no dependence on the fast variable $\tilde{z}$.

In addition, we assume that the scattered fields can be expanded in powers of $p$. That is

$$
\left.\begin{array}{rl}
\bar{E}^{s} & \sim \bar{E}^{0}(r)+\bar{e}^{0}(r, \tilde{r})+p\left[\bar{E}^{1}(r)+\bar{e}^{1}(r, \tilde{r})\right]+O\left(p^{2}\right)+\cdots \\
\bar{H}^{s} & \sim \bar{H}^{0}(r)+\bar{h}^{0}(r, \tilde{r})+p\left[\bar{H}^{1}(r)+\bar{h}^{1}(r, \tilde{r})\right]+O\left(p^{2}\right)+\cdots
\end{array}\right\}
$$

where the lower case letters, representing boundary layer vector fields, are periodic in $\tilde{y}$, are independent of $\tilde{z}$ and decay exponentially as $|\tilde{x}| \rightarrow \infty$. 


\section{MAXWELL'S EQUATIONS}

We now apply Maxwell's equations to the scattered fields and compare the coefficients of powers of $p$. Maxwell's equations for source free media are

$$
\left.\begin{array}{l}
\nabla \times \bar{E}=-j \omega \mu \bar{H} \\
\nabla \times \bar{H}=j \omega \epsilon \bar{E} .
\end{array}\right\}
$$

If we now substitute Equations (5) into these equations an use Equation (3) we get

$$
\begin{gathered}
\frac{1}{p}\left[\nabla_{\tilde{r}} \times \bar{e}^{0}(r, \tilde{r})\right]+p^{0}\left[\nabla_{r} \times \bar{E}^{0}(r)+\nabla_{\tilde{r}} \times \bar{e}^{1}(r, \tilde{r})+\nabla_{r} \times \bar{e}^{0}(r, \tilde{r})\right]+ \\
p^{1}\left[\nabla_{r} \times \bar{E}^{1}(r)+\nabla_{r} \times \bar{e}^{1}(r, \tilde{r})+\nabla_{\tilde{r}} \times \bar{e}^{2}(r, \tilde{r})\right]+O\left(p^{2}\right)+\cdots= \\
\quad-j \omega \mu\left\{p^{0}\left[\bar{H}^{0}(r)+\bar{h}^{0}(r, \tilde{r})\right]+p^{1}\left[\bar{H}^{1}(r)+\bar{h}^{1}(r, \tilde{r})\right]+O\left(p^{2}\right)+\cdots\right\}
\end{gathered}
$$

and

$$
\begin{gathered}
\frac{1}{p}\left[\nabla_{\tilde{r}} \times \bar{h}^{0}(r, \tilde{r})\right]+p^{0}\left[\nabla_{r} \times \bar{H}^{0}(r)+\nabla_{\tilde{r}} \times \bar{h}^{1}(r, \tilde{r})+\nabla_{r} \times \bar{h}^{0}(r, \tilde{r})\right]+ \\
p^{1}\left[\nabla_{r} \times \bar{H}^{1}(r)+\nabla_{r} \times \bar{h}^{1}(r, \tilde{r})+\nabla_{\tilde{r}} \times \bar{h}^{2}(r, \tilde{r})\right]+O\left(p^{2}\right)+\cdots= \\
j \omega \epsilon\left\{p^{0}\left[\bar{E}^{0}(r)+\bar{e}^{0}(r, \tilde{r})\right]+p^{1}\left[\bar{E}^{1}(r)+\bar{e}^{1}(r, \tilde{r})\right]+O\left(p^{2}\right)+\cdots\right\}
\end{gathered}
$$

The coefficients of the powers of $p$ have to be equal. We have therefore ${ }^{2}$ from the coefficients of $p^{-1}$

$$
\left.\begin{array}{rl}
\nabla_{\tilde{r}} \times \bar{e}^{0} & =0 \\
\nabla_{\tilde{r}} \times \bar{h}^{0} & =0
\end{array}\right\}
$$

the coefficients of $p^{0}$

$$
\left.\begin{array}{c}
\nabla_{\tilde{r}} \times \bar{e}^{1}=-j \omega \mu\left(\bar{H}^{0}+\bar{h}^{0}\right)-\nabla_{r} \times\left(\bar{E}^{0}+\bar{e}^{0}\right) \\
\nabla_{\tilde{r}} \times \bar{h}^{1}=j \omega \epsilon\left(\bar{E}^{0}+\bar{e}^{0}\right)-\nabla_{r} \times\left(\bar{H}^{0}+\bar{h}^{0}\right),
\end{array}\right\}
$$

and the coefficients of $p^{1}$

\footnotetext{
${ }^{2}$ Notation of specific dependencies on the slow and fast variables will be omitted unless special emphasis is needed.
} 


$$
\left.\begin{array}{l}
\nabla_{\tilde{r}} \times \bar{e}^{2}=-j \omega \mu\left(\bar{H}^{1}+\bar{h}^{1}\right)-\nabla_{r} \times\left(\bar{E}^{1}+\bar{e}^{1}\right) \\
\nabla_{\tilde{r}} \times \bar{h}^{2}=j \omega \epsilon\left(\bar{E}^{1}+\bar{e}^{1}\right)-\nabla_{r} \times\left(\bar{H}^{1}+\bar{h}^{1}\right) .
\end{array}\right\}
$$

Equations (9), (10) and (11) must now be combined and simplified. We begin by taking the divergence with respect to the fast variable of Equations (10), noting that

$$
\left.\begin{array}{c}
\nabla_{\tilde{r}} \cdot \nabla_{r} \times \bar{A}=-\nabla_{r} \cdot \nabla_{\tilde{r}} \times \bar{A} \\
\nabla_{\tilde{r}} \cdot \nabla_{\tilde{r}} \times \bar{A}=0 .
\end{array}\right\}
$$

This gives

$$
\left.\begin{array}{c}
0=-j \omega \mu \nabla_{\tilde{r}} \cdot\left(\bar{H}^{0}+\bar{h}^{0}\right)-\nabla_{\tilde{r}} \cdot \nabla_{r} \times\left(\bar{E}^{0}+\bar{e}^{0}\right) \\
0=j \omega \epsilon \nabla_{\tilde{r}} \cdot\left(\bar{E}^{0}+\bar{e}^{0}\right)-\nabla_{\tilde{r}} \cdot \nabla_{r} \times\left(\bar{H}^{0}+\bar{h}^{0}\right) .
\end{array}\right\} .
$$

Because of Equations (9), the second terms in the second parenthetical expressions of Equations (13) are zero. And, if we evaluate Equations (13) at $|\tilde{x}|=\infty$ the boundary layer terms go to zero. We then have

$$
\left.\begin{array}{c}
\nabla_{r} \times \bar{E}^{0}=-j \omega \mu \bar{H}^{0} \\
\nabla_{r} \times \bar{H}^{0}=j \omega \epsilon \bar{E}^{0}
\end{array}\right\}
$$

as expected. This gives us for Equations (13)

$$
\left.\begin{array}{l}
\nabla_{\tilde{r}} \cdot \mu \bar{h}^{0}=0 \\
\nabla_{\tilde{r}} \cdot \epsilon \bar{e}^{0}=0 .
\end{array}\right\}
$$

Applying the results of Equations (14) to Equations (10) gives

$$
\left.\begin{array}{l}
\nabla_{\tilde{r}} \times \bar{e}^{1}=-j \omega \mu \bar{h}^{0}-\nabla_{r} \times \bar{e}^{0} \\
\nabla_{\tilde{r}} \times \bar{h}^{1}=j \omega \epsilon \bar{e}^{0}-\nabla_{r} \times \bar{h}^{0} .
\end{array}\right\}
$$

If we now take the divergence of Equations (11) with respect to the fast variable and make similar arguments as above, we get

$$
\left.\begin{array}{c}
0=-j \omega \mu \nabla_{\tilde{r}} \cdot \bar{h}^{1}-\nabla_{\tilde{r}} \cdot \nabla_{r} \times \bar{e}^{-1} \\
0=j \omega \epsilon \nabla_{\tilde{r}} \cdot \bar{e}^{1}-\nabla_{\tilde{r}} \cdot \nabla_{r} \times \bar{h}^{1} .
\end{array}\right\}
$$

Combining and simplifying Equations (16) and (17) yields 


$$
\left.\begin{array}{c}
\nabla_{\tilde{r}} \cdot \bar{e}^{1}=-\nabla_{r} \cdot \bar{e}^{0} \\
\nabla_{\tilde{r}} \cdot \bar{h}^{1}=-\nabla_{r} \cdot \bar{h}^{0} .
\end{array}\right\}
$$

To recapitulate this section, the important results for the boundary layer fields are gathered below, keeping in mind that there are two equations for each result in the two different media.

$$
\begin{aligned}
& \left.\begin{array}{l}
\nabla_{\tilde{r}} \times \bar{e}^{0}=0 \\
\nabla_{\tilde{r}} \times \bar{h}^{0}=0
\end{array}\right\} \\
& \left.\begin{array}{r}
\nabla_{\tilde{r}} \cdot \epsilon \bar{e}^{0}=0 \\
\nabla_{\tilde{r}} \cdot \mu \bar{h}^{0}=0
\end{array}\right\} \\
& \left.\begin{array}{c}
\nabla_{\tilde{r}} \times \bar{e}^{1}=-j \omega \mu \bar{h}^{0}-\nabla_{r} \times \bar{e}^{0} \\
\nabla_{\tilde{r}} \times \bar{h}^{1}=j \omega \epsilon \bar{e}^{0}-\nabla_{r} \times \bar{h}^{0}
\end{array}\right\} \\
& \left.\begin{array}{c}
\nabla_{\tilde{r}} \cdot \bar{e}^{1}=-\nabla_{r} \cdot \bar{e}^{0} \\
\nabla_{\tilde{r}} \cdot \bar{h}^{1}=-\nabla_{r} \cdot \bar{h}^{0} .
\end{array}\right\}
\end{aligned}
$$

\section{BOUNDARY CONDITIONS}

We now need to apply boundary conditions at the interface of the two media. For the electric field they are

$$
\begin{gathered}
\hat{a}_{x} \times\left.\left(\bar{E}_{+}^{i}+\bar{E}_{+}^{s}-\bar{E}_{-}^{s}\right)\right|_{x=\tilde{x}=0}=0 \\
\left.\hat{a}_{x} \cdot\left[\epsilon_{+}\left(\bar{E}_{+}^{i}+\bar{E}_{+}^{s}\right)-\epsilon_{-} \bar{E}_{-}^{s}\right]\right|_{x=\tilde{x}=0}=\rho_{s}= \\
P_{s}^{0}(r)+\rho_{s}^{0}(r, \tilde{r})+p\left[P_{s}^{1}(r)+\rho_{s}^{1}(r, \tilde{r})\right]+O\left(p^{2}\right)
\end{gathered}
$$

and for the magnetic field we have 


$$
\begin{gathered}
\hat{a}_{x} \times\left.\left(\bar{H}_{+}^{i}+\bar{H}_{+}^{s}-\bar{H}_{-}^{s}\right)\right|_{x=\tilde{x}=0}=\bar{J}_{s}= \\
\bar{J}_{s}^{0}(r)+\bar{j}_{s}^{0}(r, \tilde{r})+p\left[\bar{J}_{s}^{1}(r)+\bar{j}_{s}^{1}(r, \tilde{r})\right]+O\left(p^{2}\right) \\
\left.\hat{a}_{x} \cdot\left[\mu_{+}\left(\bar{H}_{+}^{i}+\bar{H}_{+}^{s}\right)-\mu_{-} \bar{H}_{-}^{s}\right]\right|_{x=\tilde{x}=0}=0 .
\end{gathered}
$$

The subscripts $i$ and $s$ refer to incident and scattered fields respectively and $\rho_{s}$, the surface charge density, and $\bar{J}_{s}$, the surface current density, have been expanded in power series in $p$; and $\hat{a}_{u}$ represents a unit vector directed along the $u$-axis $(u=x, y$ or $z$ ). We now substitute Equations (5) into these boundary conditions and compare the coefficients of the powers of $p$. This gives us the boundary conditions on each of the field components. For the $p^{0}$ components we have

$$
\begin{aligned}
\hat{a}_{x} \times\left.\left(\bar{e}_{+}^{0}-\bar{e}_{-}^{0}\right)\right|_{x=\tilde{x}=0}= & -\hat{a}_{x} \times\left.\left(\bar{E}_{+}^{0}+\bar{E}_{+}^{i}-\bar{E}_{-}^{0}\right)\right|_{x=0} \\
\left.\hat{a}_{x} \cdot\left(\epsilon_{+} \bar{e}_{+}^{0}-\epsilon_{-} \bar{e}_{-}^{0}\right)\right|_{x=\tilde{x}=0}= & -\left.\hat{a}_{x} \cdot\left[\epsilon_{+}\left(\bar{E}_{+}^{0}+\bar{E}_{+}^{i}\right)-\epsilon_{-} \bar{E}_{-}^{0}\right]\right|_{x=0} \\
& +P_{S}^{0}+\rho_{s}^{0} \\
\hat{a}_{x} \times\left.\left(\bar{h}_{+}^{0}-\bar{h}_{-}^{0}\right)\right|_{x=\tilde{x}=0}= & -\hat{a}_{x} \times\left.\left(\bar{H}_{+}^{0}+\bar{H}_{+}^{i}-\bar{H}_{-}^{0}\right)\right|_{x=0} \\
& +\bar{J}_{s}^{0}+\bar{j}_{s}^{0} \\
\left.\hat{a}_{x} \cdot\left(\mu_{+} \bar{h}_{+}^{0}-\mu_{-} \bar{h}_{-}^{0}\right)\right|_{x=\tilde{x}=0}= & -\left.\hat{a}_{x} \cdot\left[\mu_{+}\left(\bar{H}_{+}^{0}+\bar{H}_{+}^{i}\right)-\mu_{-} \bar{H}_{-}^{0}\right]\right|_{x=0}
\end{aligned}
$$

and the $p^{1}$ components are 


$$
\begin{aligned}
\hat{a}_{x} \times\left.\left(\bar{e}_{+}^{1}-\bar{e}_{-}^{1}\right)\right|_{x=\tilde{x}=0}= & -\hat{a}_{x} \times\left.\left(\bar{E}_{+}^{1}-\bar{E}_{-}^{1}\right)\right|_{x=0} \\
\left.\hat{a}_{x} \cdot\left(\epsilon_{+} \bar{e}_{+}^{1}-\epsilon_{-} \bar{e}_{-}^{1}\right)\right|_{x=\tilde{x}=0}= & -\left.\hat{a}_{x} \cdot\left(\epsilon_{+} \bar{E}_{+}^{1}-\epsilon_{-} \bar{E}_{-}^{1}\right)\right|_{x=0} \\
& +P_{s}^{1}+\rho_{s}^{1} \\
\hat{a}_{x} \times\left.\left(\bar{h}_{+}^{1}-\bar{h}_{-}^{1}\right)\right|_{x=\tilde{x}=0}= & -\hat{a}_{x} \times\left.\left(\bar{H}_{+}^{1}-\bar{H}_{-}^{1}\right)\right|_{x=0} \\
& +\bar{J}_{s}^{1}+\bar{j}_{s}^{1} \\
\left.\hat{a}_{x} \cdot\left(\mu_{+} \bar{h}_{+}^{1}-\mu_{-} \bar{h}_{-}^{1}\right)\right|_{x=\tilde{x}=0}= & -\left.\hat{a}_{x} \cdot\left(\mu_{+} \bar{H}_{+}^{1}-\mu_{-} \bar{H}_{-}^{1}\right)\right|_{x=0} .
\end{aligned}
$$

These boundary conditions when combined with Equations (19) through (22) are statements of the boundary value problems for the zeroth order and first order boundary layer fields.

\section{THE ZEROTH ORDER FIELDS}

Let's first gather Equations (19), (20), (27), and (30) into problem statements for the zeroth order electric and magnetic fields. We have

$$
\left.\begin{array}{rl}
\nabla_{\tilde{r}} \times \bar{e}^{0} & =0 \\
\nabla_{\tilde{r}} \cdot \epsilon \bar{e}^{0} & =0 \\
\hat{a}_{x} \times\left.\left(\bar{e}_{+}^{0}-\bar{e}_{-}^{0}\right)\right|_{x=\tilde{x}=0} & =-\hat{a}_{x} \times\left.\left(\bar{E}_{+}^{0}+\bar{E}_{+}^{i}-\bar{E}_{-}^{0}\right)\right|_{x=0}
\end{array}\right\}
$$

where the first two equations of each set would have a + or - subscript depending on the medium. In Appendix A we show that both sides of each of the zeroth order boundary conditions in Equations (35) and (36) evaluate to zero. We then have, with the addition of Equations (28) and (29), the following problems for the zeroth order fields 


$$
\begin{aligned}
\nabla_{\tilde{r}} \times \bar{e}^{0}= & 0 \\
\nabla_{\tilde{r}} \cdot \epsilon \bar{e}^{0}= & 0 \\
\hat{a}_{x} \times\left.\left(\bar{e}_{+}^{0}-\bar{e}_{-}^{0}\right)\right|_{x=\tilde{x}=0}= & -\hat{a}_{x} \times\left.\left(\bar{E}_{+}^{0}+\bar{E}_{+}^{i}-\bar{E}_{-}^{0}\right)\right|_{x=0}=0 \\
\left.\hat{a}_{x} \cdot\left(\epsilon_{+} \bar{e}_{+}^{0}-\epsilon_{-} \bar{e}_{-}^{0}\right)\right|_{x=\tilde{x}=0}= & -\left.\hat{a}_{x} \cdot\left[\epsilon_{+}\left(\bar{E}_{+}^{0}+\bar{E}_{+}^{i}\right)-\epsilon_{-} \overline{\bar{E}}_{-}^{0}\right]\right|_{x=0} \\
& +P_{s}^{0}+\rho_{s}^{0} \\
\nabla_{\tilde{r}} \times \bar{h}^{0}= & 0 \\
\nabla_{\tilde{r}} \cdot \mu \bar{h}^{0}= & 0 \\
\left.\hat{a}_{x} \cdot\left(\mu_{+} \bar{h}_{+}^{0}-\mu_{-} \bar{h}_{-}^{0}\right)\right|_{x=\tilde{x}=0}= & -\left.\hat{a}_{x} \cdot\left[\mu_{+}\left(\bar{H}_{+}^{0}+\bar{H}_{+}^{i}\right)-\mu_{-} \bar{H}_{-}^{0}\right]\right|_{x=0} \\
= & 0 \\
\hat{a}_{x} \times\left.\left(\bar{h}_{+}^{0}-\bar{h}_{-}^{0}\right)\right|_{x=\tilde{x}=0}= & -\hat{a}_{x} \times\left.\left(\bar{H}_{+}^{0}+\bar{H}_{+}^{i}-\bar{H}_{-}^{0}\right)\right|_{x=0} \\
& +\bar{J}_{s}^{0}+\bar{j}_{s}^{0} .
\end{aligned}
$$

These equations can then be solved using the techniques in Appendix C. The solutions are

$$
\begin{aligned}
& h_{x \pm}^{0}=-\frac{1}{\mu_{ \pm}} \sum_{n=1}^{\infty} e^{\mp 2 n \pi \tilde{x}}\left(\mu_{a} K_{h} \vartheta_{n} \sin 2 n \pi \tilde{y}-\left.B_{x}^{0}\right|_{x=0} \varphi_{n} \cos 2 n \pi \tilde{y}\right) \\
& h_{y \pm}^{0}= \pm \frac{1}{\mu_{ \pm}} \sum_{n=1}^{\infty} e^{\mp 2 n \pi \tilde{x}}\left(\mu_{a} K_{h} \vartheta_{n} \cos 2 n \pi \tilde{y}+\left.B_{x}^{0}\right|_{x=0} \varphi_{n} \sin 2 n \pi \tilde{y}\right) \\
& e_{x \pm}^{0}= \pm \sum_{n=1}^{\infty} e^{\mp 2 n \pi \tilde{x}}\left(\frac{K_{e}}{\epsilon_{a}} \vartheta_{n} \cos 2 n \pi \tilde{y}-\left.E_{y}^{0}\right|_{x=0} \varphi_{n} \sin 2 n \pi \tilde{y}\right) \\
& e_{y \pm}^{0}=\sum_{n=1}^{\infty} e^{\mp 2 n \pi \tilde{x}}\left(\frac{K_{e}}{\epsilon_{a}} \vartheta_{n} \sin 2 n \pi \tilde{y}+\left.E_{y}^{0}\right|_{x=0} \varphi_{n} \cos 2 n \pi \tilde{y}\right)
\end{aligned}
$$

where 


$$
\begin{gathered}
\vartheta_{n}=2 \int_{0}^{b / 2 p} \frac{\cos \pi \tilde{y}^{\prime}}{\sqrt{s-\sin ^{2} \pi \tilde{y}^{\prime}}} \cos 2 n \pi \tilde{y}^{\prime} d \tilde{y}^{\prime} \\
\varphi_{n}=-4 \int_{0}^{b / 2 p} \frac{\sin \pi \tilde{y}^{\prime}}{\sqrt{s-\sin ^{2} \pi \tilde{y}^{\prime}}} \sin 2 n \pi \tilde{y}^{\prime} d \tilde{y}^{\prime} \\
\mu_{a}=\frac{2 \mu_{+} \mu_{-}}{\mu_{+}+\mu_{-}} \\
\epsilon_{a}=\frac{\epsilon_{+}+\epsilon_{-}}{2} .
\end{gathered}
$$

The current density in the $\tilde{x}=0$ plane is given by

$$
\left.\begin{array}{rl}
j_{s z}^{0}(\tilde{y})= & K_{h} \frac{\cos \pi \tilde{y}}{\sqrt{s-\sin ^{2} \pi \tilde{y}}} \\
& -\left.\frac{2}{\mu_{a}} B_{x}^{0}\right|_{x=0} \frac{\sin \pi \tilde{y}}{\sqrt{s-\sin ^{2} p i \tilde{y}}} \\
J_{s z}^{0}=K_{h}= & \left.\left(H_{y}^{i}+H_{y+}^{0}-H_{y-}^{0}\right)\right|_{x=0}
\end{array}\right\}
$$

and the charge density in the $\tilde{x}=0$ plane is given by

$$
\left.\begin{array}{rl}
\rho_{s}^{0}(\tilde{y})= & K_{e} \frac{\cos \pi \tilde{y}}{\sqrt{s-\sin ^{2} \pi \tilde{y}}} \\
& +\left.2 \epsilon_{a} E_{y}^{0}\right|_{x=0} \frac{\sin \pi \tilde{y}}{\sqrt{s-\sin ^{2} \pi \tilde{y}}} \\
P_{s}^{0}=K_{e}= & {\left.\left[\epsilon_{+}\left(E_{x}^{i}+E_{x+}^{0}\right)-E_{x-}^{0}\right]\right|_{x=0} .}
\end{array}\right\}
$$

We also have 


$$
\begin{aligned}
& s=\frac{1}{2}\left(1-\cos \frac{\pi b}{p}\right)=\sin ^{2} \frac{\pi b}{2 p} \\
& c=\frac{1}{2}\left(1+\cos \frac{\pi b}{p}\right)=\cos ^{2} \frac{\pi b}{2 p} .
\end{aligned}
$$

It is also shown in Appendix $\mathrm{C}$ that the zeroth order surface current densities in the $y$ direction were zero (as agrees with physical intuition), thus the zeroth order $z$ directed magnetic fields are continuous.

\section{THE FIRST ORDER FIELDS}

Equations (21) and (22) when combined with the boundary conditions of Equations (31) through (34) are statements of the boundary value problems for the first order fields.

$$
\left.\begin{array}{c}
\nabla_{\tilde{r}} \times \bar{e}^{1}=-j \omega \mu \bar{h}^{0}-\nabla_{r} \times \bar{e}^{0} \\
\nabla_{\tilde{r}} \times \bar{h}^{1}=j \omega \epsilon \bar{e}^{0}-\nabla_{r} \times \bar{h}^{0}
\end{array}\right\}
$$

where

$$
\begin{aligned}
\hat{a}_{x} \times\left.\left(\bar{e}_{+}^{1}-\bar{e}_{-}^{1}\right)\right|_{x=\tilde{x}=0} & =-\hat{a}_{x} \times\left.\left(\bar{E}_{+}^{1}-\bar{E}_{-}^{1}\right)\right|_{x=0} \\
\left.\hat{a}_{x} \cdot\left(\epsilon_{+} \bar{e}_{+}^{1}-\epsilon_{-} \bar{e}_{-}^{1}\right)\right|_{x=\tilde{x}=0} & =-\left.\hat{a}_{x} \cdot\left(\epsilon_{+} \bar{E}_{+}^{1}-\epsilon_{-} \bar{E}_{-}^{1}\right)\right|_{x=0}+P_{s}^{1}+\rho_{s}^{1}(48 \\
\hat{a}_{x} \times\left.\left(\bar{h}_{+}^{1}-\bar{h}_{-}^{1}\right)\right|_{x=\tilde{x}=0} & =-\hat{a}_{x} \times\left.\left(\bar{H}_{+}^{1}-\bar{H}_{-}^{1}\right)\right|_{x=0}+\bar{J}_{s}^{1}+\bar{j}_{s}^{1} \\
\left.\hat{a}_{x} \cdot\left(\mu_{+} \bar{h}_{+}^{1}-\mu_{-} \bar{h}_{-}^{1}\right)\right|_{x=\tilde{x}=0} & =-\left.\hat{a}_{x} \cdot\left(\mu_{+} \bar{H}_{+}^{1}-\mu_{-} \bar{H}_{-}^{1}\right)\right|_{x=0}
\end{aligned}
$$

In Appendix B we show that both sides of each of the first order boundary conditions in Equations (47) and (50) evaluate to zero. We then have the following problems for the first order fields 


$$
\left.\begin{array}{rl}
\nabla_{\tilde{r}} \times \bar{e}^{1} & =-j \omega \mu \bar{h}^{0}-\nabla_{r} \times \bar{e}^{0} \\
\nabla_{\tilde{r}} \cdot \bar{e}^{1} & =-\nabla_{r} \cdot \bar{e}^{0} \\
\hat{a}_{x} \times\left.\left(\bar{e}_{+}^{1}-\bar{e}_{-}^{1}\right)\right|_{x=\tilde{x}=0} & =-\hat{a}_{x} \times\left.\left(\bar{E}_{+}^{1}-\bar{E}_{-}^{1}\right)\right|_{x=\tilde{x}=0}=0 \\
\left.\hat{a}_{x} \cdot\left(\epsilon_{+} \bar{e}_{+}^{1}-\epsilon_{-} \bar{e}_{-}^{1}\right)\right|_{x=\tilde{x}=0} & =-\left.\hat{a}_{x} \cdot\left(\epsilon_{+} \bar{E}_{+}^{1}-\epsilon_{-} \bar{E}_{-}^{1}\right)\right|_{x=0}+P_{s}^{1}+\rho_{s}^{1}
\end{array}\right\}
$$

$$
\left.\begin{array}{rl}
\nabla_{\tilde{r}} \times \bar{h}^{1} & =j \omega \epsilon \bar{e}^{0}-\nabla_{r} \times \bar{h}^{0} \\
\nabla_{\tilde{r}} \cdot \bar{h}^{1} & =-\nabla_{r} \cdot \bar{h}^{0} \\
\left.\hat{a}_{x} \cdot\left(\mu_{+} \bar{h}_{+}^{1}-\mu_{-} \bar{h}_{-}^{1}\right)\right|_{x=\tilde{x}=0} & =-\left.\hat{a}_{x} \cdot\left(\mu_{+} \bar{H}_{+}^{1}-\mu_{-} \bar{H}_{-}^{1}\right)\right|_{x=\tilde{x}=0}=0 \\
\hat{a}_{x} \times\left.\left(\bar{h}_{+}^{1}-\bar{h}_{-}^{1}\right)\right|_{x=\tilde{x}=0} & =-\hat{a}_{x} \times\left.\left(\bar{H}_{+}^{1}-\bar{H}_{-}^{1}\right)\right|_{x=0}+\bar{J}_{s}^{1}+\bar{j}_{s}^{1} .
\end{array}\right\}
$$

We take a direct approach to solving Equations (51) and (52) by expanding the first of them while noting that $\frac{\partial}{\partial \tilde{z}}=0, h_{z}^{0}=0$ and $e_{z}^{0}=0$. This gives

$$
\left.\begin{array}{rl}
\hat{a}_{x} \frac{\partial e_{z}^{1}}{\partial \tilde{y}}-\hat{a}_{y} \frac{\partial e_{z}^{1}}{\partial \tilde{x}}+\hat{a}_{z}\left(\frac{\partial e_{y}^{1}}{\partial \tilde{x}}-\frac{\partial e_{x}^{1}}{\partial \tilde{y}}\right)=-j \omega \mu\left(\hat{a}_{x} h_{x}^{0}+\hat{a}_{y} h_{y}^{0}\right) \\
+\hat{a}_{x} \frac{\partial e_{y}^{0}}{\partial z}-\hat{a}_{y} \frac{\partial e_{x}^{0}}{\partial z}-\hat{a}_{z}\left(\frac{\partial e_{y}^{0}}{\partial x}-\frac{\partial e_{x}^{0}}{\partial y}\right) \\
\hat{a}_{x} \frac{\partial h_{z}^{1}}{\partial \tilde{y}}-\hat{a}_{y} \frac{\partial h_{z}^{1}}{\partial \tilde{x}}+\hat{a}_{z}\left(\frac{\partial h_{y}^{1}}{\partial \tilde{x}}-\frac{\partial h_{x}^{1}}{\partial \tilde{y}}\right)=j \omega \epsilon\left(\hat{a}_{x} e_{x}^{0}+\hat{a}_{y} e_{y}^{0}\right) \\
+\hat{a}_{x} \frac{\partial h_{y}^{0}}{\partial z}-\hat{a}_{y} \frac{\partial h_{x}^{0}}{\partial z}-\hat{a}_{z}\left(\frac{\partial h_{y}^{0}}{\partial x}-\frac{\partial h_{x}^{0}}{\partial y}\right) .
\end{array}\right\}
$$

Equating the $\hat{a}_{x}$ and $\hat{a}_{y}$ components of each equation yields 


$$
\left.\begin{array}{l}
\frac{\partial e_{z}^{1}}{\partial \tilde{y}}=-j \omega \mu h_{x}^{0}+\frac{\partial e_{y}^{0}}{\partial z} \\
\frac{\partial h_{z}^{1}}{\partial \tilde{y}}=j \omega \epsilon e_{x}^{0}+\frac{\partial h_{y}^{0}}{\partial z}
\end{array}\right\}
$$

and

$$
\left.\begin{array}{l}
\frac{\partial e_{z}^{1}}{\partial \tilde{x}_{1}}=j \omega \mu h_{y}^{0}+\frac{\partial e_{x}^{0}}{\partial z} \\
\frac{\partial h_{z}^{1}}{\partial \tilde{x}}=-j \omega \epsilon e_{y}^{0}+\frac{\partial h_{x}^{0}}{\partial z} .
\end{array}\right\}
$$

We can find $e_{z}^{1}$ and $h_{z}^{1}$ by integrating Equation (54) or (55) with respect to $\tilde{y}$ or $\tilde{x}$ respectively. The easiest method is to integrate Equations (55) over $\tilde{x}^{\prime}$ from $\pm \infty$ to $\tilde{x}$. This gives, after substitution of Equations (39)

$$
\left.\begin{array}{rl}
e_{z \pm}^{1} & =-j \omega \sum_{n=1}^{\infty} \frac{e^{\mp 2 n \pi \tilde{x}}}{2 n \pi}\left(\mu_{a} K_{h} \vartheta_{n} \cos 2 n \pi \tilde{y}+\left.B_{x}^{0}\right|_{x=0} \varphi_{n} \sin 2 n \pi \tilde{y}\right) \\
& -\sum_{n=1}^{\infty} \frac{e^{\mp 2 n \pi \tilde{x}}}{2 n \pi}\left(\frac{1}{\epsilon_{a}} \frac{\partial K_{e}}{\partial z} \vartheta_{n} \cos 2 n \pi \tilde{y}-\frac{\left.\partial E_{y}^{0}\right|_{x=0}}{\partial z} \varphi_{n} \sin 2 n \pi \tilde{y}\right) \\
h_{z \pm}^{1} & = \pm j \omega \epsilon_{ \pm} \sum_{n=1}^{\infty} \frac{e^{\mp 2 n \pi \tilde{x}}}{2 n \pi}\left(\frac{K_{e}}{\epsilon_{a}} \vartheta_{n} \sin 2 n \pi \tilde{y}+\left.E_{y}^{0}\right|_{x=0} \varphi_{n} \cos 2 n \pi \tilde{y}\right) \\
& \pm \frac{1}{\mu_{ \pm}} \sum_{n=1}^{\infty} \frac{e^{\mp 2 n \pi \tilde{x}}}{2 n \pi}\left(\mu_{a} \frac{\partial K_{h}}{\partial z} \vartheta_{n} \sin 2 n \pi \tilde{y}-\frac{\left.\partial B_{x}^{0}\right|_{x=0}}{\partial z} \varphi_{n} \cos 2 n \pi \tilde{y}\right) .
\end{array}\right\}
$$

These are the first order fields of interest since they will give the averaged boundary conditions for the total $z$ directed fields.

\section{EQUIVALENT BOUNDARY CONDITIONS}

Now we evaluate the boundary conditions on the fields of Equations (56) noting that $\left.e_{z+}^{1}\right|_{\tilde{x}=0}=\left.\left.e_{z-}^{1}\right|_{\tilde{x}=0} \equiv e_{z}^{1}\right|_{\tilde{x}=0}$. This gives, after some rearrangement 


$$
\left.\begin{array}{rl}
\left.e_{z}^{1}\right|_{\tilde{x}=0} & =-\left[\left.j \omega B_{x}^{0}\right|_{x=0}-\frac{\left.\partial E_{y}^{0}\right|_{x=0}}{\partial z}\right] \sum_{n=1}^{\infty} \frac{\varphi_{n}}{2 n \pi} \sin 2 n \pi \tilde{y} \\
& -\left[j \omega \mu_{a} K_{h}+\frac{1}{\epsilon_{a}} \frac{\partial K_{e}}{\partial z}\right] \sum_{n=1}^{\infty} \frac{\vartheta_{n}}{2 n \pi} \cos 2 n \pi \tilde{y} \\
\left.\left(h_{z+}^{1}-h_{z-}^{1}\right)\right|_{\tilde{x}=0} & =2\left[j \omega K_{e}+\frac{\partial K_{h}}{\partial z}\right] \sum_{n=1}^{\infty} \frac{\vartheta_{n}}{2 n \pi} \sin 2 n \pi \tilde{y} \\
& +2\left[\left.j \omega \epsilon_{a} E_{y}^{0}\right|_{x=0}-\frac{1}{\mu_{a}} \frac{\left.\partial B_{x}^{0}\right|_{x=0}}{\partial z}\right] \sum_{n=1}^{\infty} \frac{\varphi_{n}}{2 n \pi} \cos 2 n \pi \tilde{y} .
\end{array}\right\}
$$

We need to evaluate these equations either on the strip or in the gap in order to find the boundary conditions on the slow variable, first order fields.

\subsection{Equivalent boundary conditions - electric field.}

We first evaluate the first of Equations (57) on the strip. Look at the first bracketed term of this expression. We know from the $x$ component of the first of Equations (14) that

$$
\frac{\partial E_{z}^{0}}{\partial y}-\frac{\partial E_{y}^{0}}{\partial z}=-j \omega B_{x}^{0} .
$$

Since we have shown that $e_{z}^{0} \equiv 0$ and, on the strip, $e_{z}^{0}=-E_{z}^{0}$ we have

$$
\frac{\partial E_{y}^{0}}{\partial z}=j \omega B_{x}^{0}
$$

If we now also note that $e_{z}^{1}=-E_{z}^{1}$ on the strip, we have

$$
\left.E_{z}^{1}\right|_{x=0}=\left[j \omega \mu_{a} K_{h}+\frac{1}{\epsilon_{a}} \frac{\partial K_{e}}{\partial z}\right] \sum_{n=1}^{\infty} \frac{\vartheta_{n}}{2 n \pi} \cos 2 n \pi \tilde{y} .
$$

Since $\left.E_{z}^{1}\right|_{x=0}$ is a function of $y$ and $z$ alone, the right hand side of Equation (60) should also be a function of $y$ and $z$ alone. This means that the summation must be a constant. In order to evaluate the summation, we must rely on some information from the solution of the zeroth order fields. From Equations (176) and (185), we have on the strip 


$$
\Phi=\left.E_{y}^{0}\right|_{x=0} \tilde{y}+\Phi_{0}=\sum_{n=1}^{\infty}\left(k_{n} \cos 2 n \pi \tilde{y}+l_{n} \sin 2 n \pi \tilde{y}\right) .
$$

The even part of this equation with respect to $\tilde{y}$ is

$$
\Phi_{0}=\sum_{n=1}^{\infty} k_{n} \cos 2 n \pi \tilde{y}
$$

From Equations (199) and (200) we have

$$
k_{n}=\frac{K_{e}}{\epsilon_{a}} \frac{\vartheta_{n}}{2 n \pi} .
$$

Substitute Equation (63) into (62) to get

$$
\Phi_{0}=\frac{K_{e}}{\epsilon_{a}} \sum_{n=1}^{\infty} \frac{\vartheta_{n}}{2 n \pi} \cos 2 n \pi \tilde{y} .
$$

But from Equation (190) we have that

$$
\Phi_{0}=-\frac{\ln s}{4 \pi \epsilon_{a}} K_{e}
$$

Combining Equations (64) and (65) gives

$$
\sum_{n=1}^{\infty} \frac{\vartheta_{n}}{2 n \pi} \cos 2 n \pi \tilde{y}=-\frac{\ln s}{4 \pi} .
$$

Substituting this result into Equation (60) gives

$$
\left.E_{z}^{1}\right|_{x=0}=-\frac{\ln s}{4 \pi}\left[j \omega \mu_{a} K_{h}+\frac{1}{\epsilon_{a}} \frac{\partial K_{e}}{\partial z}\right] .
$$

The average fields are given by

$$
\left.\begin{array}{rl}
\bar{E}_{a v} & =\int_{0}^{1} \bar{E}^{t} d \tilde{y} \\
& =\int_{0}^{1}\left[\left(\bar{E}^{i}+\bar{E}^{o}\right)+\bar{e}^{o}+p\left(\bar{E}^{1}+\bar{e}^{1}\right)+O\left(p^{2}\right)\right] d \tilde{y} .
\end{array}\right\}
$$

All of the average values are contained in the slow variable fields, and the average of the fast variable fields over a unit cell is zero (Appendix B). The integration then gives 


$$
\bar{E}_{a v}=\left(\bar{E}^{i}+\bar{E}^{o}\right)+p \bar{E}^{1} .
$$

We are interested in the average boundary conditions on the $z$ directed fields. Since the zeroth order fields are continuous, the only contribution to the boundary conditions on the average fields are the first order and higher fields. So, to first order we have from Equations (67) and (69) that

$$
\left.E_{z}\right|_{x=0}=\left.p E_{z}^{1}\right|_{x=0}=-\frac{p \ln s}{4 \pi}\left[j \omega \mu_{a} K_{h}+\frac{1}{\epsilon_{a}} \frac{\partial K_{e}}{\partial z}\right] .
$$

Substituting the values for $K_{e}$ and $K_{h}$ gives our desired equivalent boundary condition for the $z$ directed electric field.

$$
\left.E_{z}\right|_{x=0}=-\frac{p \ln s}{4 \pi}\left[\left.j \omega \mu_{a}\left(H_{y}^{+}-H_{y}^{-}\right)\right|_{x=0}+\left.\frac{1}{\epsilon_{a}} \frac{\partial}{\partial z}\left(\epsilon_{+} E_{x}^{+}-\epsilon_{-} E_{x}^{-}\right)\right|_{x=0}\right] .
$$

\subsection{Equivalent boundary conditions - magnetic field.}

We now need to evaluate the second of Equations (57) in the gap. From the last of Equations (52) we have that

$$
j_{s y}^{1}=-J_{s y}^{1}-\left.\left(H_{z+}^{1}-H_{z-}^{1}\right)\right|_{x=0}-\left.\left(h_{z+}^{1}-h_{z-}^{1}\right)\right|_{x=\tilde{x}=0} .
$$

The average value of $j_{s y}^{1}$ is zero so that

$$
J_{s y}^{1}=-\left.\left(H_{z+}^{1}-H_{z-}^{1}\right)\right|_{x=0}
$$

and

$$
\left.\begin{array}{rl}
j_{s y}^{1}= & -\left.\left(h_{z+}^{1}-h_{z-}^{1}\right)\right|_{x=\tilde{x}=0} \\
= & -2\left[j \omega K_{e}+\frac{\partial K_{h}}{\partial z}\right] \sum_{n=1}^{\infty} \frac{\vartheta_{n}}{2 n \pi} \sin 2 n \pi \tilde{y} \\
& -2\left[\left.j \omega \epsilon_{a} E_{y}^{0}\right|_{x=0}-\frac{1}{\mu_{a}} \frac{\left.\partial B_{x}^{0}\right|_{x=0}}{\partial z}\right] \sum_{n=1}^{\infty} \frac{\varphi_{n}}{2 n \pi} \cos 2 n \pi \tilde{y} .
\end{array}\right\}
$$


In the gap, $j_{s y}^{1}+J_{s y}^{1}=0$ or $j_{s y}^{1}=-J_{s y}^{1}=\left.\left(H_{z+}^{1}-H_{z-}^{1}\right)\right|_{x=0}$. We also know that $K_{e}$ and $K_{h}$ both evaluate to zero in the gap. We then have

$$
\left.\left(H_{z+}^{1}-H_{z-}^{1}\right)\right|_{x=0}=-\left.2\left[j \omega \epsilon_{a} E_{y}^{0}-\frac{1}{\mu_{a}} \frac{\partial B_{x}^{0}}{\partial z}\right] \sum_{n=1}^{\infty} \frac{\varphi_{n}}{2 n \pi} \cos 2 n \pi \tilde{y}\right|_{x=0} .
$$

Since $\left.\left(H_{z+}^{1}-H_{z-}^{1}\right)\right|_{x=0}$ must be a constant with respect to the fast variable, the summation on the right hand side of Equation (75) must be a constant. If we substitute the value of $\varphi_{n}$ from Equation (40) into the summation of Equation (75) we get

$$
\sum_{n=1}^{\infty} \frac{\varphi_{n}}{2 n \pi} \cos 2 n \pi \tilde{y}=-\frac{2}{\pi} \int_{0}^{b / 2 p} \frac{\sin \pi \tilde{y}^{\prime}}{\sqrt{s-\sin ^{2} \pi \tilde{y}^{\prime}}} \sum_{n=1}^{\infty} \frac{1}{n} \sin 2 n \pi \tilde{y}^{\prime} \cos 2 n \pi \tilde{y} d \tilde{y}^{\prime} .
$$

If we use the identity of Equation (157) and

$$
\sum_{n=1}^{\infty} \frac{1}{n} \sin \zeta=\frac{1}{2}(\pi-\zeta), \text { for } 0<\zeta<2 \pi
$$

and the fact that we are evaluating Equation (76) in the gap where $b / 2 p<$ $\tilde{y}<1 / 2$ we have that

$$
\begin{aligned}
\sum_{n=1}^{\infty} \frac{1}{n} \sin 2 n \pi \tilde{y}^{\prime} \cos 2 n \pi \tilde{y} & =\frac{1}{4}\left[\pi-2 \pi\left(\tilde{y}^{\prime}+\tilde{y}\right)\right]-\frac{1}{4}\left[\pi-2 \pi\left(\tilde{y}-\tilde{y}^{\prime}\right)\right] \\
& =-\pi \tilde{y}^{\prime} .
\end{aligned}
$$

We then have for our summation

$$
\sum_{n=1}^{\infty} \frac{\varphi_{n}}{2 n \pi} \cos 2 n \pi \tilde{y}=2 \int_{0}^{b / 2 p} \frac{\sin \pi \tilde{y}^{\prime}}{\sqrt{s-\sin ^{2} \pi \tilde{y}^{\prime}}} \tilde{y}^{\prime} d \tilde{y}^{\prime} .
$$

Only a simple change of variable remains to put the integral into the form of an integral found in [23] and reproduced here for convenience 


$$
\int_{0}^{\beta} \frac{x \sin x d x}{\left(1-\sin ^{2} \alpha \sin ^{2} x\right) \sqrt{\sin ^{2} \alpha-\sin ^{2} x}}=\frac{\pi \ln \frac{\cos \alpha+\sqrt{1-\sin ^{2} \alpha \sin ^{2} \beta}}{2 \cos \beta \cos ^{2} \frac{\alpha}{2}}}{2 \cos \alpha \sqrt{1-\sin ^{2} \alpha \sin ^{2} \beta}} .
$$

Evaluation of Equation (80) with $\alpha=0$ and $\beta=\frac{\pi b}{2 p}$, further manipulation, and substitution into Equation (79) gives

$$
\sum_{n=1}^{\infty} \frac{\varphi_{n}}{2 n \pi} \cos 2 n \pi \tilde{y}=-\frac{1}{2 \pi} \ln c .
$$

So the result for the boundary condition for the first order $z$ directed magnetic fields is

$$
\left.\left(H_{z+}^{1}-H_{z-}^{1}\right)\right|_{x=0}=\frac{1}{\pi} \ln c\left[j \omega \epsilon_{a} E_{y}^{0}-\frac{1}{\mu_{a}} \frac{\partial B_{x}^{0}}{\partial z}\right]_{x=0} .
$$

Using the same arguments as for the electric field boundary conditons as in the previous section, we have to first order

$$
\left.\left(H_{z+}-H_{z-}\right)\right|_{x=0}=\frac{p}{\pi} \ln c\left[j \omega \epsilon_{a} E_{y}^{0}-\frac{1}{\mu_{a}} \frac{\partial B_{x}^{0}}{\partial z}\right]_{x=0} .
$$

\section{RESULTS AND CONCLUSIONS}

From [15] the equivalent boundary conditions are $E_{z}, E_{y}$, and $B_{x}$ are continuous, and ${ }^{3}$

$$
\begin{aligned}
E_{z}= & \frac{l_{2}}{2}\left[j \omega \mu_{a}\left(H_{y}^{+}-H_{y}^{-}\right)+\frac{1}{\epsilon_{a}} \frac{\partial}{\partial z}\left(\epsilon_{+} E_{x}^{+}-\epsilon_{-} E_{x}^{-}\right)\right] \\
& H_{z}^{+}-H_{z}^{-}=2 l_{1}\left(-j \omega \epsilon_{a} E_{y}+\frac{1}{\mu_{a}} \frac{\partial B_{x}}{\partial z}\right)
\end{aligned}
$$

where

\footnotetext{
${ }^{3}$ All of the following equations of boundary conditions assume evaluation in the $x=0$ plane.
} 


$$
\left.\begin{array}{l}
l_{1}=-\frac{p}{2 \pi} \ln c=\frac{p}{\pi} \ln \sec \frac{\pi b}{2 p}=\frac{p}{\pi} \ln \csc \frac{\pi a}{2 p} \\
l_{2}=-\frac{p}{2 \pi} \ln s=\frac{p}{\pi} \ln \csc \frac{\pi b}{2 p}=\frac{p}{\pi} \ln \sec \frac{\pi a}{2 p}
\end{array}\right\}
$$

and

$$
\left.\begin{array}{rl}
\mu_{a} & =\frac{2 \mu_{+} \mu_{-}}{\mu_{+}+\mu_{-}} \\
\epsilon_{a} & =\frac{\epsilon_{+}+\epsilon_{-}}{2}
\end{array}\right\}
$$

Comparison of these equations with the results of the previous sections show that our results (to first order) are equivalent to those of [15]. However, the use of homogenization techniques shows explicitly that the "equivalent boundary conditions" or "averaged boundary conditions" refered to in [15] as well as other references cited in this report, are the boundary conditions of the average, non-boundary layer fields.

From [24] the equivalent boundary conditions are $E_{z}, E_{y}$, and $B_{x}$ are continuous, and

$$
\left.\begin{array}{c}
E_{z}=\frac{l_{2}}{2}\left[j \omega \mu_{a}\left(H_{y}^{+}-H_{y}^{-}\right)+\frac{j}{\omega \epsilon_{a}} \frac{\partial^{2}}{\partial z^{2}}\left(H_{y}^{+}-H_{y}^{-}\right)\right] \\
H_{z}^{+}-H_{z}^{-}=2 l_{1}\left(-j \omega \epsilon_{a} E_{y}-\frac{j}{\omega \mu_{a}} \frac{\partial^{2} E y}{\partial z^{2}}\right)
\end{array}\right\}
$$

which, by the use of Maxwell's equations can be transformed into

$$
\begin{aligned}
E_{z}= & \frac{l_{2}}{2}\left[j \omega \mu_{a}\left(H_{y}^{+}-H_{y}^{-}\right)+\frac{1}{\epsilon_{a}} \frac{\partial}{\partial z}\left(\epsilon_{+} E_{x}^{+}-\epsilon_{-} E_{x}^{-}\right)\right. \\
& \left.+\frac{j}{\omega \epsilon_{a}} \frac{\partial^{2}}{\partial z \partial y}\left(H_{z}^{+}-H_{z}^{-}\right)\right] \\
H_{z}^{+}-H_{z}^{-}= & 2 l_{1}\left(-j \omega \epsilon_{a} E_{y}+\frac{1}{\mu_{a}} \frac{\partial B_{x}}{\partial z}-\frac{j}{\omega \mu_{a}} \frac{\partial^{2} E_{z}}{\partial z \partial y}\right)
\end{aligned}
$$


The last terms of these expressions are second order results, which presumabley could be derived from the solution of our second order equations. This is the advantage to using homogenization techniques; i.e., given enough time and mathematical persistence, solutions to the desired order can be derived. It is not clear that Equation (88) contains all of the second order terms that would be found in this way.

In conclusion, an independent verification of the results of [10], [11], [13], [15] and, to first order, [24] has been made. Our technique of homogenization relies on a relatively basic level of mathematics found in most engineering mathematics textbooks, and some specific solutions to integral equations found in [17]. In addition, the use of this technique allows a look at the nature of the charge and current distributions on the grid and the resulting structure of the boundary layer fields. Such insight into these fields has, in general, not been provided by methods used in the above references. Finally, a clear picture of the origin of the resulting boundary conditions of the average fields is obtained. It is now straightforward to apply this technique to the derivation of equivalent boundary conditions for more general types of structure.

Having obtained these boundary conditions and confirmed their proper form, the next step will be to use them in an integral equation formulation for the solution of boundary problems involving finely slotted microstrip patch structures. This will be taken up in subsequent work. 


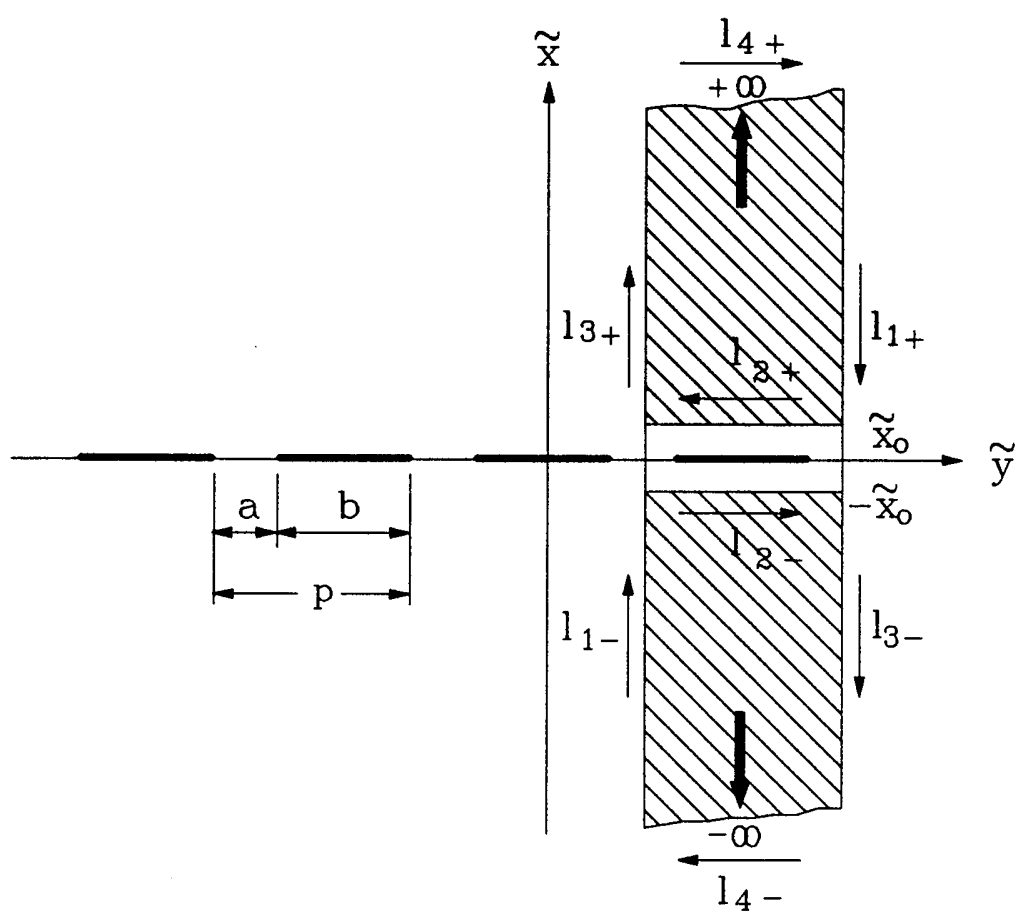

Figure 2: Surface of cross-section of a unit cell.

\section{A ZEROTH ORDER BOUNDARY CONDITIONS}

\section{A.1 Introduction}

Our goal here is to simplify the boundary value problems presented by Equations (35) and (36). In order to do this we need to look at the third of these equations with the intent of integrating those fields over the surface of a "unit cell," that is, the surface whose cross-section has width $y=p(\tilde{y}=1)$ and extends to $\tilde{x} \rightarrow \pm \infty$ as shown in Figure 2 .

We can represent $\bar{h}^{0}$ and $\bar{e}^{0}$ as the sum of components transverse and normal to this surface. That is 


$$
\bar{h}^{0}=\bar{h}_{t}^{0}+\hat{a}_{z} h_{z}^{0} \text { and } \bar{e}^{0}=\bar{e}_{t}^{0}+\hat{a}_{z} e_{z}^{0}
$$

where

$$
\bar{h}_{t}^{0}=\hat{a}_{x} h_{x}^{0}+\hat{a}_{y} h_{y}^{0} \text { and } \bar{e}_{t}^{0}=\hat{a}_{x} e_{x}^{0}+\hat{a}_{y} e_{y}^{0} .
$$

If we now substitute these vectors into Equations (9) we get

$$
\left.\begin{array}{c}
\nabla_{\tilde{r}} \times \bar{e}^{0}=\nabla_{\tilde{r}} \times \bar{e}_{t}^{0}+\nabla_{\tilde{r}} \times \hat{a}_{z} e_{z}^{0}=0 \\
\nabla_{\tilde{r}} \times \bar{h}^{0}=\nabla_{\tilde{r}} \times \bar{h}_{t}^{0}+\nabla_{\tilde{r}} \times \hat{a}_{z} h_{z}^{0}=0 .
\end{array}\right\}
$$

Using the vector identity

$$
\nabla \times(\bar{A} \psi)=\nabla \psi \times \bar{A}+\psi \nabla \times \bar{A}
$$

on the second terms of Equations (89), and noting that the first term yields a vector in the $\hat{a}_{z}$ direction gives

$$
\left.\begin{array}{r}
\nabla_{\tilde{r}} \times \bar{e}^{0}=\hat{a}_{z}\left(\hat{a}_{z} \cdot \nabla_{\tilde{r}} \times \bar{e}_{t}^{0}\right)-\hat{a}_{z} \times \nabla_{\tilde{r}} e_{z}^{0}=0 \\
\nabla_{\tilde{r}} \times \bar{h}^{0}=\hat{a}_{z}\left(\hat{a}_{z} \cdot \nabla_{\tilde{r}} \times \bar{h}_{t}^{0}\right)-\hat{a}_{z} \times \nabla_{\tilde{r}} h_{z}^{0}=0 .
\end{array}\right\}
$$

From the transverse part of these equations, we have that

$$
\left.\begin{array}{l}
\hat{a}_{z} \times \nabla_{\tilde{r}} e_{z}^{0}=0 \text { or } \nabla_{\tilde{r}} e_{z}^{0}=0 \\
\hat{a}_{z} \times \nabla_{\tilde{r}} h_{z}^{0}=0 \text { or } \nabla_{\tilde{r}} h_{z}^{0}=0
\end{array}\right\}
$$

so that these vector components must be equal to a constant with respect to the fast variable. But since they must also decay to zero at $\tilde{x}= \pm \infty$, we must have

$$
e_{z}^{0}=h_{z}^{0}=0
$$

and, from the third of Equations (35), the sum of the $z$ components of the slow variable electric fields must also be zero, that is, the $z$ components of these fields are continuous. 
To determine the transverse components of the fields, we must resort to another method for each of the fields separately.

\section{A.2 The Electric Field Boundary Conditions}

If we substitute the first of Equations (92) into the first of Equations (89), we get

$$
\left(\nabla_{\tilde{r}} \times \bar{e}_{t}^{0}\right) \cdot \hat{a}_{z}=0
$$

We can integrate this over the top half of the unit cell, call it $S_{+}\left(\tilde{x}_{0}\right)$, of Figure 2. This surface is chosen so that $\tilde{x}_{0}$ can be any value including zero. We have

$$
\int_{S_{+}\left(\tilde{x}_{0}\right)}\left(\nabla_{\tilde{r}} \times \vec{e}_{t+}^{0}\right) \cdot \tilde{a}_{z} d S_{+}=0 .
$$

We now use Stokes' Theorem to transform this surface integral to a line integral around the surface. The line segments are shown in Figure 2 with the total given by $l_{+}\left(\tilde{x}_{0}\right)$. We have

$$
\int_{S_{+}\left(\tilde{x}_{0}\right)}\left(\nabla_{\tilde{r}} \times \bar{e}_{t+}^{0}\right) \cdot \tilde{a}_{z} d S_{+}=\oint_{l_{+}\left(\tilde{x}_{0}\right)} \bar{e}_{t+}^{0} \cdot d \bar{l}_{+} .
$$

Because of periodicity, the segment of the integral labeled $l_{1+}$ is equal to the negative of the segment labeled $l_{3+}$. Also, the segment labeled $l_{4+}$ is zero because the field component $\bar{e}_{t+}^{0}$ is zero at $\infty$. The only segment left is that labeled $l_{2+}$ so that

$$
\int_{S_{+}\left(\tilde{x}_{0}\right)}\left(\nabla_{\tilde{r}} \times \bar{e}_{t+}^{0}\right) \cdot \tilde{a}_{z} d S_{+}=\int_{l_{2+}\left(\tilde{x}_{0}\right)} \bar{e}_{t+}^{0} \cdot d \bar{l}_{2+}=-\int_{1}^{0} \bar{e}_{t+}^{0} \cdot \hat{a}_{y} d \tilde{y}=0 .
$$

A similar integration over the bottom half of the surface $\left(S_{-}\left(\tilde{x}_{0}\right)\right)$ gives

$$
\int_{S_{-}\left(\tilde{x}_{0}\right)}\left(\nabla_{\tilde{r}} \times \bar{e}_{t-}^{0}\right) \cdot \tilde{a}_{z} d S_{-}=\int_{l_{2-}\left(\tilde{x}_{0}\right)} \bar{e}_{t-}^{0} \cdot d \bar{l}_{2-}=\int_{0}^{1} \bar{e}_{t-}^{0} \cdot \hat{a}_{y} d \tilde{y}=0 .
$$

Equations (97) and (98) are true for any value of $\tilde{x}_{0}$ and they also happen

to be the average values of $e_{y}^{0}$ over a unit cell for any value of $\tilde{x}_{0}$. We can also make arguments as above to show that (using the second of Equations (35)) 


$$
\int_{S_{+}\left(\tilde{x}_{0}\right)} \nabla_{\tilde{r}} \cdot\left(\epsilon_{+} \vec{e}_{t}^{0}\right) d \tilde{S}_{+}=\int_{1}^{0} \epsilon_{+} \vec{e}_{t}^{0} \cdot \hat{a}_{x} d \tilde{y}=0
$$

with a similar equation for $S_{-}\left(\tilde{x}_{0}\right)$. Thus, the average values of $e_{x}^{0}$ over a unit cell are also zero. This is to say that the "average" fields are precisely $\bar{E}^{0}$ and do not involve the boundary layer fields.

If we now combine Equations (97) and (98) taken at $\tilde{x}_{0}=0$, we have

$$
\left.\int_{0}^{1}\left(\bar{e}_{t+}^{0}-\bar{e}_{t-}^{0}\right)\right|_{x=\tilde{x}=0} \cdot \hat{a}_{y} d \tilde{y}=0 .
$$

From the third of Equations (35), we also have

$$
\left.\int_{0}^{1}\left(\bar{E}_{t+}^{0}+\bar{E}_{t+}^{i}-\bar{E}_{t-}^{0}\right)\right|_{x=0} \cdot \hat{a}_{y} d \tilde{y}=0 .
$$

But, these fields are constant with respect to $\tilde{y}$ so we conclude that

$$
\left.\left(\bar{E}_{t+}^{0}+\bar{E}_{t+}^{i}-\bar{E}_{t-}^{0}\right)\right|_{x=0} \cdot \hat{a}_{y}=0 .
$$

This fact leads to the deduction that the zeroth order, slow variable tangential electric fields at the interface are continuous. The third of Equations (35) can then be written

$$
\hat{a}_{x} \times\left.\left(\bar{e}_{+}^{0}-\bar{e}_{-}^{0}\right)\right|_{x=\tilde{x}=0}=0
$$

so that our final conclusion is that these fields also have continuous components tangential to the grating.

\section{A.3 The Magnetic Field Boundary Conditions}

If we integrate the second of Equations (36) over $S_{+}\left(\tilde{x}_{0}\right)$, we have by the divergence theorem

$$
\int_{S_{+}\left(\tilde{x}_{0}\right)} \nabla_{\tilde{r}} \cdot \mu_{+} \bar{h}_{t+}^{0} d S_{+}=\oint_{l_{+}\left(\tilde{x}_{0}\right)} \mu_{+} \bar{h}_{t+}^{0} \cdot \hat{a}_{n} d l_{+}=0
$$

where $\hat{a}_{n}$ is the outward normal to the contour $l_{+}\left(\tilde{x}_{0}\right)$. Again, the integrals over $l_{1+}$ and $l_{3+}$ cancel and $l_{4+}$ go to zero so that we are left with 


$$
\int_{S_{+}\left(\tilde{x}_{0}\right)} \nabla_{\tilde{r}} \cdot \mu_{+} \bar{h}_{t+}^{0} d S_{+}=\int_{1}^{0} \mu_{+} \bar{h}_{t+}^{0} \cdot \hat{a}_{x} d \tilde{y}=0 .
$$

A similar integration over $S_{-}\left(\tilde{x}_{0}\right)$ gives

$$
\int_{S_{-}\left(\tilde{x}_{0}\right)} \nabla_{\tilde{r}} \cdot \mu_{-} \bar{h}_{t-}^{0} d S_{-}=\int_{0}^{1} \mu_{-} \bar{h}_{t-}^{0} \cdot \hat{a}_{x} d \tilde{y}=0 .
$$

Once again, this shows that the average of the $h_{x}^{0}$ field over a unit cell is zero for any value of $\tilde{x}_{0}$. We can also show that (using the first of Equations $(36))$

$$
\int_{S_{+}\left(\tilde{x}_{0}\right)}\left(\nabla_{\tilde{r}} \times \bar{h}_{t+}^{0}\right) \cdot \tilde{a}_{z} d S_{+}=-\int_{1}^{0} \bar{h}_{t+}^{0} \cdot \hat{a}_{y} d \tilde{y}=0
$$

so that the average of $h_{y}^{0}$ over a unit cell is also zero for any $\tilde{x}_{0}$ and that the "average" fields are precisely $\bar{H}^{0}$.

Taking $\tilde{x}_{0}=0$ and combining Equations (105), (106) and (30) leads to

$$
\left.\int_{p}^{2 p}\left[\mu_{+}\left(\bar{H}_{t+}^{0}+\bar{H}_{t+}^{i}\right)-\mu_{-} \bar{H}_{t-}^{0}\right]\right|_{x=0} \cdot \hat{a}_{x} d \tilde{y}=0 .
$$

Again, these fields are constant with respect to $\tilde{y}$ so that we come to the conclusion that

$$
\left.\left[\mu_{+}\left(\bar{H}_{t+}^{0}+\bar{H}_{t+}^{i}\right)-\mu_{-} \bar{H}_{t-}^{0}\right]\right|_{x=0} \cdot \hat{a}_{x}=0
$$

and the third of Equations (36) can be revised accordingly. The conslusion here is that the normal components of the magnetic flux densities of the zeroth order fields are continuous.

\section{B FIRST ORDER BOUNDARY CONDITIONS}

We can simplify the first order boundary conditions and also gain some further insight into the final outcome of the averaged boundary conditions. We begin with Equations (21) and Equations (22) 


$$
\begin{gathered}
\nabla_{\tilde{r}} \times \bar{e}^{1}=-j \omega \mu \bar{h}^{0}-\nabla_{r} \times \bar{e}^{0} \\
\nabla_{\tilde{r}} \times \bar{h}^{1}=j \omega \epsilon \bar{e}^{0}-\nabla_{r} \times \bar{h}^{0} \\
\nabla_{\tilde{r}} \cdot \bar{e}^{1}=-\nabla_{r} \cdot \bar{e}^{0} \\
\nabla_{\tilde{r}} \cdot \bar{h}^{1}=-\nabla_{r} \cdot \bar{h}^{0} .
\end{gathered}
$$

We can integrate these equations over the top surface of a unit cell as depicted in Figure 2. This gives

$$
\left.\begin{array}{rl}
\int_{S_{+}\left(\tilde{x}_{0}\right)}\left(\nabla_{\tilde{r}} \times \bar{e}^{1}\right) \cdot \hat{a}_{z} d \tilde{S}_{+}= & -j \omega \mu_{+} \int_{S_{+}\left(\tilde{x}_{0}\right)} \bar{h}^{0} \cdot \hat{a}_{z} d \tilde{S}_{+} \\
& -\nabla_{r} \times \int_{S_{+}\left(\tilde{x}_{0}\right)} \bar{e}^{0} \cdot \hat{a}_{z} d \tilde{S}_{+} \\
\int_{S_{+}\left(\tilde{x}_{0}\right)}\left(\nabla_{\tilde{r}} \times \bar{h}^{1}\right) \cdot \hat{a}_{z} d \tilde{S}_{+}= & j \omega \epsilon_{+} \int_{S_{+}\left(\tilde{x}_{0}\right)} \bar{e}^{0} \cdot \hat{a}_{z} d \tilde{S}_{+} \\
& -\nabla_{r} \times \int_{S_{+}\left(\tilde{x}_{0}\right)}^{h^{0} \cdot \hat{a}_{z} d \tilde{S}_{+}} \\
\epsilon_{+} \int_{S_{+}\left(\tilde{x}_{0}\right)} \nabla_{\tilde{r}} \cdot \bar{e}^{1} d \tilde{S}_{+}= & -\epsilon_{+} \nabla_{r} \cdot \int_{S_{+}\left(\tilde{x}_{0}\right)}^{\bar{e}^{0} d \tilde{S}_{+}} \\
\mu_{+} \int_{S_{+}\left(\tilde{x}_{0}\right)} \nabla_{\tilde{r}} \cdot \bar{h}^{1} d \tilde{S}_{+}= & -\mu_{+} \nabla_{r} \cdot \int_{S_{+}\left(\tilde{x}_{0}\right)}^{h^{0} d \tilde{S}_{+}}
\end{array}\right\}
$$

Since integration is over the fast variable, the right hand side of Equations (111), after expansion, will integrate to zero because of the periodic fields and the fact that $e_{z}^{0}=h_{z}^{0}=0$. Integration over the lower half of the unit cell produces similar results. Since the integration is valid for all values of $\tilde{x}_{0}$ and we can transform the left hand sides of Equations (111) to line integrals as in the previous section, we can make the same conclusions as that section for the first order boundary layer fields. That is, that their average values are zero. We can continue this process to show that all boundary layer fields have average values of zero and that the "average" fields are precisely the slow variable fields. 
Following the derivation of Appendix A using Stokes' theorem and the divergence theorem we can show that the terms on both sides of Equations (31) and (34) go to zero. This brings us to the conclusion that the first order electric fields tangent to the grating and the first order magnetic flux densities normal to the grating are also continuous and that Equations (31) and (34) can be modified accordingly.

\section{THE STATIC PROBLEMS}

\section{C.1 Introduction}

The general approach for the solution of Equations (37) and (38) is to describe the fields in terms of potentials which in turn are expanded in Fourier series in the $\tilde{y}$ variable. Advantage is taken of the expected exponential decay in the $\tilde{x}$ direction and the choice of the direction of the vector potential is facilitated by the independence of the fields from $\tilde{z}$ and the fact that $e_{z}^{0}$ and $h_{z}^{0}$ are zero.

\section{C.2 The static problem for the magnetic field.}

For the magnetic field problem, we define a vector potential $\bar{A}$ such that

$$
\mu \bar{h}^{0}=\nabla_{\tilde{r}} \times \bar{A}
$$

where $\bar{A}(\tilde{x}, \tilde{y})=A_{z}(\tilde{x}, \tilde{y}) \hat{a}_{z}$ so that

$$
h_{y}^{0}=-\frac{1}{\mu} \frac{\partial A_{z}}{\partial \tilde{x}} \text { and } h_{x}^{0}=\frac{1}{\mu} \frac{\partial A_{z}}{\partial \tilde{y}} .
$$

Because $\bar{A}(\tilde{x}, \tilde{y})$ is periodic with period 1 , we can expand $A_{z}(\tilde{x}, \tilde{y})$ in a Fourier series such as

$$
A_{z}(\tilde{x}, \tilde{y})=\frac{a_{0}(\tilde{x})}{2}+\sum_{n=1}^{\infty} a_{n}(\tilde{x}) \cos 2 n \pi \tilde{y}+\sum_{n=1}^{\infty} b_{n}(\tilde{x}) \sin 2 n \pi \tilde{y}
$$

where 


$$
\left.\begin{array}{c}
a_{n}(\tilde{x})=2 \int_{-1 / 2}^{1 / 2} A_{z}\left(\tilde{x}, \tilde{y}^{\prime}\right) \cos 2 n \pi \tilde{y}^{\prime} d \tilde{y}^{\prime}, n=0,1,2, \ldots \\
b_{n}(\tilde{x})=2 \int_{-1 / 2}^{1 / 2} A_{z}\left(\tilde{x}, \tilde{y}^{\prime}\right) \sin 2 n \pi \tilde{y}^{\prime} d \tilde{y}^{\prime}, n=1,2, \ldots
\end{array}\right\}
$$

If we take the curl of Equation (112) we get

$$
\nabla_{\tilde{r}} \times \mu \bar{h}^{0}=\nabla_{\tilde{r}} \times \nabla_{\tilde{r}} \times \bar{A}=0 .
$$

Using a vector identity for the vector triple product, we have

$$
\nabla_{\tilde{r}}\left(\nabla_{\tilde{r}} \cdot \bar{A}\right)-\nabla_{\tilde{r}}^{2} \bar{A}=0 .
$$

Since $\bar{A}=A_{z}(\tilde{x}, \tilde{y}) \hat{a}_{z}, \nabla_{\tilde{r}} \cdot \bar{A}=0$, and we have

$$
\nabla_{\tilde{r}}^{2} \bar{A}=0
$$

or

$$
\frac{\partial^{2} A_{z}}{\partial \tilde{x}^{2}}+\frac{\partial^{2} A_{z}}{\partial \tilde{y}^{2}}=0 .
$$

We now substitute Equation (114) into Equation (116) to get (after comparing coefficients of the sine and cosine terms)

$$
\frac{\partial^{2} a_{0}(\tilde{x})}{\partial \tilde{x}^{2}}=0
$$

and

$$
\left.\begin{array}{l}
\frac{\partial^{2} a_{n}(\tilde{x})}{\partial \tilde{x}^{2}}-4 n^{2} \pi^{2} a_{n}(\tilde{x})=0 \\
\frac{\partial^{2} b_{n}(\tilde{x})}{\partial \tilde{x}^{2}}-4 n^{2} \pi^{2} b_{n}(\tilde{x})=0
\end{array}\right\}
$$

The solutions to Equations (118) on either side of the grid are given by 


$$
\begin{aligned}
& a_{n+}(\tilde{x})=g_{1 n} e^{-2 n \pi \tilde{x}}, \text { and } b_{n+}(\tilde{x})=f_{1 n} e^{-2 n \pi \tilde{x}}, \tilde{x}>0 \\
& a_{n-}(\tilde{x})=g_{2 n} e^{+2 n \pi \tilde{x}}, \text { and } b_{n-}(\tilde{x})=f_{2 n} e^{+2 n \pi \tilde{x}}, \tilde{x}<0 .
\end{aligned}
$$

The general solution to Equation (117) is a linear equation in $\tilde{x}$. We require that the fields go to zero as $|\tilde{x}|$ goes to $\infty$ so that from Equations (113), $a_{0}(\tilde{x})=$ a constant. We call this constant $2 A_{0}$, so that

$$
\left.\begin{array}{l}
A_{z+}(\tilde{x}, \tilde{y})=A_{0}+\sum_{n=1}^{\infty} e^{-2 n \pi \tilde{x}}\left(g_{1 n} \cos 2 n \pi \tilde{y}+f_{1 n} \sin 2 n \pi \tilde{y}\right), \tilde{x}>0 \\
A_{z-}(\tilde{x}, \tilde{y})=A_{0}+\sum_{n=1}^{\infty} e^{+2 n \pi \tilde{x}}\left(g_{2 n} \cos 2 n \pi \tilde{y}+f_{2 n} \sin 2 n \pi \tilde{y}\right), \tilde{x}<0
\end{array}\right\}
$$

We can then substitute Equations (121) into Equations (113) to get the fields. Also, since the $x$ components of the magnetic flux densities, $\mu h_{x}^{0}$, are continuous at $x=0, g_{1 n}=g_{2 n} \equiv g_{n}$ and $f_{1 n}=f_{2 n} \equiv f_{n}$. This in turn shows that $\bar{A}$ is continuous at $\tilde{x}=0$. We then have

$$
\left.\begin{array}{ll}
h_{x+}^{0}=-\frac{1}{\mu_{+}} \sum_{n=1}^{\infty} 2 n \pi e^{-2 n \pi \tilde{x}}\left(g_{n} \sin 2 n \pi \tilde{y}-f_{n} \cos 2 n \pi \tilde{y}\right), & \tilde{x}>0 \\
h_{x-}^{0}=-\frac{1}{\mu_{-}} \sum_{n=1}^{\infty} 2 n \pi e^{+2 n \pi \tilde{x}}\left(g_{n} \sin 2 n \pi \tilde{y}-f_{n} \cos 2 n \pi \tilde{y}\right), & \tilde{x}<0 \\
h_{y+}^{0}=\frac{1}{\mu_{+}} \sum_{n=1}^{\infty} 2 n \pi e^{-2 n \pi \tilde{x}}\left(g_{n} \cos 2 n \pi \tilde{y}+f_{n} \sin 2 n \pi \tilde{y}\right), & \tilde{x}>0 \\
h_{y-}^{0}=-\frac{1}{\mu_{-}} \sum_{n=1}^{\infty} 2 n \pi e^{+2 n \pi \tilde{x}}\left(g_{n} \cos 2 n \pi \tilde{y}+f_{n} \sin 2 n \pi \tilde{y}\right), & \tilde{x}<0 .
\end{array}\right\}
$$

From the fourth of Equation (38) we have on the strip 


$$
\left.\begin{array}{c}
\left.\left(H_{y}^{i}+H_{y+}^{0}+h_{y+}^{0}-H_{y-}^{0}-h_{y-}^{0}\right)\right|_{x=\tilde{x}=0}=J_{s z}^{0}+j_{s z}^{0} \\
\left.\left(H_{z}^{i}+H_{z+}^{0}+h_{z+}^{0}-H_{z-}^{0}-h_{z-}^{0}\right)\right|_{x=\tilde{x}=0}=-J_{s y}^{0}-j_{s y}^{0} .
\end{array}\right\}
$$

We have shown that $h_{z}^{0}=0$. Therefore, since the left hand side of the second of Equations (123) is a function of $y$ and $z$ only, the right hand side must also be a function or $y$ and $z$ only. We then conclude that $j_{s y}^{0}$ is a function of $y$ and $z$ alone. Since $J_{s y}^{0}+j_{s y}^{0}$ is zero in the gap and doesn't depend on $\tilde{y}$, we conclude that the right hand side of the second of Equations (123) is identically zero. The contribution from the slow variable magnetic fields is a constant with respect to the fast variable. Let us put $\left.\left(H_{y}^{i}+H_{y+}^{0}-H_{y-}^{0}\right)\right|_{x=0}=$ $K_{h}$, where it is understood that $K_{h}$ is a function of $y$ and $z$. We then have for Equations (123)

$$
\left.\begin{array}{c}
\left.\left(h_{y+}^{0}-h_{y-}^{0}\right)\right|_{x=\tilde{x}=0}+K_{h}=J_{s z}^{0}+j_{s z}^{0} \\
\left.\left(H_{z}^{i}+H_{z+}^{0}-H_{z-}^{0}\right)\right|_{x=0}=0
\end{array}\right\}
$$

We now substitute the second two of Equations (122) into the first of Equations (124). This gives

$$
j_{s z}^{0}=K_{h}-J_{s z}^{0}+\left(\frac{1}{\mu_{+}}+\frac{1}{\mu_{-}}\right) \sum_{n=1}^{\infty} 2 n \pi\left(g_{n} \cos 2 n \pi \tilde{y}+f_{n} \sin 2 n \pi \tilde{y}\right) .
$$

The right hand side of Equation (125) is a Fourier series representation of $j_{s z}^{0}$ with coefficients given by

$$
\left.\begin{array}{l}
K_{h}-J_{s z}^{0}=\int_{-1 / 2}^{1 / 2} j_{s z}^{0}\left(\tilde{y}^{\prime}\right) d \tilde{y}^{\prime} \\
n \pi=2 \int_{-1 / 2}^{1 / 2} j_{s z}^{0}\left(\tilde{y}^{\prime}\right) \cos 2 n \pi \tilde{y}^{\prime} d \tilde{y}^{\prime}, \quad n=1,2, \ldots \\
n \pi=2 \int_{-1 / 2}^{1 / 2} j_{s z}^{0}\left(\tilde{y}^{\prime}\right) \sin 2 n \pi \tilde{y}^{\prime} d \tilde{y}^{\prime}, \quad n=1,2, \ldots
\end{array}\right\}
$$

We note here that the first of Equations (126) gives the average value of $j_{s z}^{0}(\tilde{y})$, which is zero, so that $K_{h}=J_{s z}^{0}$. In the gap, we have that $J_{s z}^{0}+j_{s z}^{0}=0$, or $j_{s z}^{0}=-J_{s z}^{0}$ so we can split the integrals of Equations (126) into those in the gap and those on the strip. After a little algebra we have 


$$
\left.\begin{array}{c}
K_{h}-J_{s z}^{0} \frac{b}{p}=\int_{-b / 2 p}^{b / 2 p} j_{s z}^{0}\left(\tilde{y}^{\prime}\right) d \tilde{y}^{\prime} \\
g_{n}=\frac{\mu_{a}}{2 n \pi} \int_{-b / 2 p}^{b / 2 p}\left[J_{s z}^{0}+j_{s z}^{0}\left(\tilde{y}^{\prime}\right)\right] \cos 2 n \pi \tilde{y}^{\prime} d \tilde{y}^{\prime} \\
f_{n}=\frac{\mu_{a}}{2 n \pi} \int_{-b / 2 p}^{b / 2 p} j_{s z}^{0}\left(\tilde{y}^{\prime}\right) \sin 2 n \pi \tilde{y}^{\prime} d \tilde{y}^{\prime}
\end{array}\right\}
$$

where

$$
\mu_{a}=\frac{2 \mu_{+} \mu_{-}}{\mu_{+}+\mu_{-}}
$$

and use has been made of the fact that

$$
-\int_{b / 2 p}^{1 / 2} J_{s z}^{0} \cos 2 n \pi \tilde{y}^{\prime} d \tilde{y}^{\prime}=\int_{0}^{b / 2 p} J_{s z}^{0} \cos 2 n \pi \tilde{y}^{\prime} d \tilde{y}^{\prime}
$$

Now, $h_{x}^{0}+\left.H_{x}^{0}\right|_{x=0}=0$ or $h_{x}^{0}=-\left.H_{x}^{0}\right|_{x=0}$ on the strip. ${ }^{4}$ From Equation (113) we must have on the $\operatorname{strip}(\tilde{x}=0)$ :

$$
\frac{1}{\mu} \frac{\partial A_{z}}{\partial \tilde{y}}=-\left.H_{x}^{0}\right|_{x=0}
$$

Since $\left.H_{x}^{0}\right|_{x=0}$ is a function of $y$ and $z$ alone, we can integrate to get

$$
A_{z}=-\left.\mu H_{x}^{0}\right|_{x=0} \tilde{y}+A_{1}
$$

where $A_{1}$ is a constant with respect to $\tilde{r}$ and $\mu H_{x}^{0}(r)=B_{x}^{0}(r)$ is continuous at $x=0$. We now substitute Equations (127) and (131) into Equations (121) evaluated at $\tilde{x}=0$ to arrive at

$$
\left.\begin{array}{rl}
A_{2}-\left.B_{x}^{0}\right|_{x=0} \tilde{y}= & \sum_{n=1}^{\infty} \frac{\mu_{a}}{2 n \pi} \cos 2 n \pi \tilde{y} \int_{-b / 2 p}^{b / 2 p}\left[J_{s z}^{0}+j_{s z}^{0}\left(\tilde{y}^{\prime}\right)\right] \cos 2 n \pi \tilde{y}^{\prime} d \tilde{y}^{\prime} \\
& +\sum_{n=1}^{\infty} \frac{\mu_{a}}{2 n \pi} \sin 2 n \pi \tilde{y} \int_{-b / 2 p}^{b / 2 p} j_{s z}^{0}\left(\tilde{y}^{\prime}\right) \sin 2 n \pi \tilde{y}^{\prime} d \tilde{y}^{\prime}
\end{array}\right\}
$$

where

\footnotetext{
${ }^{4}$ Here, the total zeroth order magnetic field includes the incident field and since it is evaluated at $x=0$, is a function of $y$ and $z$.
} 


$$
A_{2}=A_{1}-A_{0} .
$$

After rearranging and switching the order of integration and summation we have

$$
\left.\begin{array}{rl}
\frac{2 \pi}{\mu_{a}}\left[A_{2}-\left.B_{x}^{0}\right|_{x=0} \tilde{y}\right]= & \int_{-b / 2 p}^{b / 2 p}\left[J_{s z}^{0}+j_{s z}^{0}\left(\tilde{y}^{\prime}\right)\right] \sum_{n=1}^{\infty} \frac{1}{n} \cos 2 n \pi \tilde{y} \cos 2 n \pi \tilde{y}^{\prime} d \tilde{y}^{\prime} \\
& +\int_{-b / 2 p}^{b / 2 p} j_{s z}^{0}\left(\tilde{y}^{\prime}\right) \sum_{n=1}^{\infty} \frac{1}{n} \sin 2 n \pi \tilde{y} \sin 2 n \pi \tilde{y}^{\prime} d \tilde{y}^{\prime}
\end{array}\right\}
$$

Equation (134) is an integral equation for $j_{s z}^{0}(\tilde{y})$. Before we continue though, we need to make some observations as to the difficulties in solving this equation as it stands. First, our limits are not over a full period of the grid, meaning that the sine and cosine functions are not orthoganal over this interval. We will deal with this problem later with a change of variable. Also, even though our limits on the integrals are symmetric, we have made no assumptions as to the symmetry of $j_{s z}^{0}(\tilde{y})$. Indeed, it must be assumed that it has no symmetry. To make our problem simpler, we can separate $j_{s z}^{0}(\tilde{y})$ into even and odd parts by the following identity:

$$
\left.\begin{array}{rl}
j_{s z}^{0}(\tilde{y}) & =\frac{1}{2}\left[j_{s z}^{0}(\tilde{y})+j_{s z}^{0}(-\tilde{y})\right]+\frac{1}{2}\left[j_{s z}^{0}(\tilde{y})-j_{s z}^{0}(-\tilde{y})\right] \\
& \equiv \frac{1}{2} j_{s z}^{0 e}\left(\tilde{y}^{\prime}\right)+\frac{1}{2} j_{s z}^{0 o}\left(\tilde{y}^{\prime}\right)
\end{array}\right\}
$$

where the first bracketed term is an even function (defined as $j_{s z}^{0 e}$ ) and the second bracketed term is an odd function (defined as $j_{s z}^{00}$ ).

We can now look at Equation (134) as being composed of an even part and an odd part. On the left, the even part is the constant term and the odd part the remaining term in $\tilde{y}$. Substitution of Equation (135) into the right hand side of Equation (134) simplifies it considerably because integration of an odd function over symmetric limits yields zero and the integration over the resulting even functions can be transformed into twice the integration over half of the segment. Once this is done, we can separate Equation (134) into its even and odd parts to yield two separate integral equations for solution. They are: 


$$
\left.\begin{array}{rl}
\frac{2 \pi}{\mu_{a}} A_{2} & =\int_{0}^{b / 2 p} \mathcal{J}_{s z}^{0 e}\left(y, z, \tilde{y}^{\prime}\right) \sum_{n=1}^{\infty} \frac{1}{n} \cos 2 n \pi \tilde{y} \cos 2 n \pi \tilde{y}^{\prime} d \tilde{y}^{\prime} \\
-\left.\frac{2 \pi}{\mu_{a}} B_{x}^{0}\right|_{x=0} \tilde{y} & =\int_{0}^{b / 2 p} j_{s z}^{0 o}\left(\tilde{y}^{\prime}\right) \sum_{n=1}^{\infty} \frac{1}{n} \sin 2 n \pi \tilde{y} \sin 2 n \pi \tilde{y}^{\prime} d \tilde{y}^{\prime} .
\end{array}\right\}
$$

where

$$
\mathcal{J}_{s z}^{0 e}\left(y, z, \tilde{y}^{\prime}\right)=2 J_{s z}^{0}(y, z)+j_{s z}^{0 e}(\tilde{y}) .
$$

The solution for $j_{s z}^{0}(\tilde{y})$ will be one half of the sum of the solutions for Equations (136).

\section{C.2.1 The even current function.}

The solution of the first of Equations (136) follows that of Lewin in [17]. First look at the summation of the first of Equations (136). We can use the identities

$$
\begin{aligned}
\sin \eta \sin \xi & =\frac{1}{2}[\cos (\eta-\xi)-\cos (\eta+\xi)] \\
\cos \eta \cos \xi & =\frac{1}{2}[\cos (\eta+\xi)+\cos (\eta-\xi)] \\
\sum_{n=1}^{\infty} \frac{\cos n \zeta}{n} & =-\ln \left|2 \sin \frac{\zeta}{2}\right|, 0<\zeta<2 \pi
\end{aligned}
$$

to transform the summation of the first of Equations (136) to

$$
\sum_{n=1}^{\infty} \frac{1}{n} \cos 2 n \pi \tilde{y} \cos 2 n \pi \tilde{y}^{\prime}=-\frac{1}{2} \ln \left(2\left|\cos 2 \pi \tilde{y}-\cos 2 \pi \tilde{y}^{\prime}\right|\right) .
$$

Now we use the transformation due to Schwinger [25]. Let

$$
\left.\begin{array}{r}
\cos 2 \pi \tilde{y}=c+s \cos \theta \\
\cos 2 \pi \tilde{y}^{\prime}=c+s \cos \theta^{\prime} .
\end{array}\right\}
$$

For $\tilde{y}=0$, we want $\theta=0$, and for $\tilde{y}=b / 2 p$, we want $\theta=\pi$. Substituting these values into Equations (142) and solving for $s$ and $c$ yields 


$$
\begin{aligned}
& s=\frac{1}{2}\left(1-\cos \frac{\pi b}{p}\right)=\sin ^{2} \frac{\pi b}{2 p} \\
& c=\frac{1}{2}\left(1+\cos \frac{\pi b}{p}\right)=\cos ^{2} \frac{\pi b}{2 p} .
\end{aligned}
$$

If we now substitute Equations (142) into Equation (141) we get

$$
\sum_{n=1}^{\infty} \frac{1}{n} \cos 2 n \pi \tilde{y} \cos 2 n \pi \tilde{y}^{\prime}=-\frac{1}{2} \ln s-\frac{1}{2} \ln \left(2\left|\cos \theta-\cos \theta^{\prime}\right|\right) .
$$

We can use the form of Equation (141) to expand $\ln \left(2\left|\cos \theta-\cos \theta^{\prime}\right|\right)$ back into a cosine series. The result is

$$
\sum_{n=1}^{\infty} \frac{1}{n} \cos 2 n \pi \tilde{y} \cos 2 n \pi \tilde{y}^{\prime}=-\frac{1}{2} \ln s+\sum_{n=1}^{\infty} \frac{1}{n} \cos n \theta \cos n \theta^{\prime} .
$$

Substituting this result into the first of Equations (136) with the associated changes in integration limits and noting that

$$
\mathcal{J}_{s z}^{0 e}\left(y, z, \tilde{y}^{\prime}\right) d \tilde{y}=\mathcal{J}_{s z}^{0 e}\left(y, z, \tilde{y}^{\prime}\right) \frac{d \tilde{y}^{\prime}}{d \theta^{\prime}} d \theta^{\prime}
$$

we have

$$
\left.\begin{array}{rl}
\frac{2 \pi}{\mu_{a}} A_{2} & =-\frac{1}{2} \ln s \int_{0}^{\pi} \mathcal{J}_{s z}^{0 e}\left(y, z, \tilde{y}^{\prime}\right) \frac{d \tilde{y}^{\prime}}{d \theta^{\prime}} d \theta^{\prime} \\
& +\int_{0}^{\pi} \mathcal{J}_{s z}^{0 e}\left(y, z, \tilde{y}^{\prime}\right) \frac{d \tilde{y}^{\prime}}{d \theta^{\prime}} \sum_{n=1}^{\infty} \frac{1}{n} \cos n \theta \cos n \theta^{\prime} d \theta^{\prime} .
\end{array}\right\}
$$

Now the summation on the right is orthogonal over the interval 0 to $\pi$ and can be reshaped into a Fourier cosine series. The constant on the left must be equal to the constant term on the right hand side. Likewise, the coefficients of $\cos n \theta$ on the right must equal the corresponding terms on the left hand side which are all zero. Equating these terms gives 


$$
\left.\begin{array}{c}
\frac{2 \pi}{\mu_{a}} A_{2}=-\frac{1}{2} \ln s \int_{0}^{\pi} \mathcal{J}_{s z}^{0 e}\left(y, z, \tilde{y}^{\prime}\right) \frac{d \tilde{y}^{\prime}}{d \theta^{\prime}} d \theta^{\prime} \\
0=\int_{0}^{\pi} \mathcal{J}_{s z}^{0 e}\left(y, z, \tilde{y}^{\prime}\right) \frac{d \tilde{y}^{\prime}}{d \theta^{\prime}} \frac{\cos n \theta^{\prime}}{n} d \theta^{\prime} .
\end{array}\right\}
$$

We can rearrange the first of Equations (147) to get

$$
-\frac{4}{\mu_{a}} \frac{A_{2}}{\ln s}=\frac{1}{\pi} \int_{0}^{\pi} \mathcal{J}_{s z}^{0 e}\left(y, z, \tilde{y}^{\prime}\right) \frac{d \tilde{y}^{\prime}}{d \theta^{\prime}} d \theta^{\prime} .
$$

But, the right hand side is just the constant term for the Fourier cosine representation of the function $\mathcal{J}_{s z}^{0 e}(y, z, \tilde{y}) \frac{d \tilde{y}}{d \theta}$. That is, since there are no $\cos n \theta$ terms

$$
\mathcal{J}_{s z}^{0 e}(y, z, \tilde{y}) \frac{d \tilde{y}}{d \theta}=-\frac{4}{\mu_{a}} \frac{A_{2}}{\ln s} .
$$

But, from Equations (142) we have that

$$
\left.\begin{array}{c}
\frac{d \tilde{y}}{d \theta}=\frac{1}{2 \pi} \frac{s \sin \theta}{\sin 2 \pi \tilde{y}} \\
s \sin \theta=\sqrt{s^{2}-(\cos 2 \pi \tilde{y}-c)^{2}} .
\end{array}\right\}
$$

If we now substitute Equations (150) into Equation (149) we have

$$
\mathcal{J}_{s z}^{0 e}(y, z, \tilde{y})=-\frac{8 \pi}{\mu_{a}} \frac{A_{2}}{\ln s} \frac{\sin 2 \pi \tilde{y}}{\sqrt{s^{2}-(\cos 2 \pi \tilde{y}-c)^{2}}}
$$

After further manipulation we have

$$
\mathcal{J}_{s z}^{0 e}(y, z, \tilde{y})=-\frac{8 \pi}{\mu_{a}} \frac{A_{2}}{\ln s} \frac{\cos \pi \tilde{y}}{\sqrt{s-\sin ^{2} \pi \tilde{y}}} .
$$

We now apply Equations (137) to get

$$
2 J_{s z}^{0}+j_{s z}^{0 e}(\tilde{y})=-\frac{8 \pi}{\mu_{a}} \frac{A_{2}}{\ln s} \frac{\cos \pi \tilde{y}}{\sqrt{s-\sin ^{2} \pi \tilde{y}}}
$$

\section{C.2.2 The odd current function.}

We now proceed to the second of Equations (136) 


$$
-\left.\frac{2 \pi}{\mu_{a}} B_{x}^{0}\right|_{x=0} \tilde{y}=\int_{0}^{b / 2 p} j_{s z}^{0 o}\left(\tilde{y}^{\prime}\right) \sum_{n=1}^{\infty} \frac{1}{n} \sin 2 n \pi \tilde{y} \sin 2 n \pi \tilde{y}^{\prime} d \tilde{y}^{\prime} .
$$

We can use the identities of Equations (138) and (140) to change the summation to a more suitable closed form. We then have

$$
\left.\frac{-4 \pi}{\mu_{a}} B_{x}^{0}\right|_{x=0} \tilde{y}=\int_{0}^{b / 2 p} j_{s z}^{0 o}\left(\tilde{y}^{\prime}\right)\left[\ln \left|2 \sin \pi\left(\tilde{y}+\tilde{y}^{\prime}\right)\right|-\ln \left|2 \sin \pi\left(\tilde{y}-\tilde{y}^{\prime}\right)\right|\right] d \tilde{y}^{\prime}
$$

Now, we differentiate with respect to $\tilde{y}$ to get

$$
-\left.\frac{4}{\mu_{a}} B_{x}^{0}\right|_{x=0}=\mathrm{PV} \int_{0}^{b / 2 p} j_{s z}^{0 o}\left(\tilde{y}^{\prime}\right)\left[\frac{\cos \pi\left(\tilde{y}+\tilde{y}^{\prime}\right)}{\sin \pi\left(\tilde{y}+\tilde{y}^{\prime}\right)}-\frac{\cos \pi\left(\tilde{y}-\tilde{y}^{\prime}\right)}{\sin \pi\left(\tilde{y}-\tilde{y}^{\prime}\right)}\right] d \tilde{y}^{\prime} .
$$

where the integral is taken in the Cauchy principal value sense.

Further manipulation using the identity of Equation (138) and the identity

$$
\sin \eta \cos \xi=\frac{1}{2}[\sin (\eta-\xi)+\sin (\eta+\xi)]
$$

yields

$$
-\left.\frac{2}{\mu_{a}} B_{x}^{0}\right|_{x=0}=\int_{0}^{b / 2 p} j_{s z}^{0 o}\left(\tilde{y}^{\prime}\right) \frac{\sin 2 \pi \tilde{y}^{\prime}}{\cos 2 \pi \tilde{y}-\cos 2 \pi \tilde{y}^{\prime}} d \tilde{y}^{\prime} .
$$

We now use the Schwinger transformations with the modification

$$
\left.\begin{array}{rl}
\cos 2 \pi \tilde{y} & =c+s v \\
\cos 2 \pi \tilde{y}^{\prime} & =c+s u
\end{array}\right\}
$$

This time, we want $u=v=1$ for $\tilde{y}=\tilde{y}^{\prime}=0$ and $u=v=-1$ for $\tilde{y}=\tilde{y}^{\prime}=b / 2 p$. This choice gives us values for $c$ and $s$ as before given by Equations (143) and (144). We also note that

$$
\sin 2 \pi \tilde{y}^{\prime} d \tilde{y}^{\prime}=-\frac{s}{2 \pi} d u
$$

Making the substitutions of Equations (159) and (160) into Equation (158) gives 


$$
\left.\frac{4}{\mu_{a}} B_{x}^{0}\right|_{x=0}=\frac{1}{\pi} \int_{-1}^{1} \frac{j_{s z}^{0 o}(u)}{u-v} d u
$$

Integral equations of this form are discussed in [17]. The solution of the equation is given by

$$
j_{s z}^{0 o}(v)=\frac{C_{j}+\left.\frac{4}{\mu_{a}} B_{x}^{0}\right|_{x=0} v}{\sqrt{1-v^{2}}}
$$

where $C_{j}$ is an arbitrary constant. From the first of Equations (159),

$$
v=\frac{1}{s}(\cos 2 \pi \tilde{y}-c)
$$

so that we have

$$
j_{s z}^{0 o}(\tilde{y})=\frac{C_{j} s+\left.\frac{4}{\mu_{a}} B_{x}^{0}\right|_{x=0}(\cos 2 \pi \tilde{y}-c)}{\sqrt{s^{2}-(\cos 2 \pi \tilde{y}-c)^{2}}} .
$$

We need to look at the behavior of this function as $\tilde{y} \rightarrow 0$. We can easily see that we have a singularity at $\tilde{y}=0$ which is not physically reasonable. To eliminate this singularity, we set the numerator of Equation (164) to 0 at $\tilde{y}=0$. This requires that

$$
C_{j}=-\left.\frac{4}{\mu_{a}} B_{x}^{0}\right|_{x=0}
$$

We then have

$$
j_{s z}^{0 o}(\tilde{y})=\left.\frac{4}{\mu_{a}} B_{x}^{0}\right|_{x=0} \frac{\cos 2 \pi \tilde{y}-1}{\sqrt{s^{2}-(\cos 2 \pi \tilde{y}-c)^{2}}} .
$$

Further manipulation yields

$$
j_{s z}^{0 o}(\tilde{y})=-\left.\frac{4}{\mu_{a}} B_{x}^{0}\right|_{x=0} \frac{\sin \pi \tilde{y}}{\sqrt{s-\sin ^{2} \pi \tilde{y}}} .
$$

\section{C.2.3 The surface current.}

Finally, if we take one half of the sum of Equations (153) and (167) we have the current density 


$$
\left.\begin{array}{rl}
j_{s z}^{0}(\tilde{y})= & -\frac{4 \pi}{\mu_{a}} \frac{A_{2}}{\ln s} \frac{\cos \pi \tilde{y}}{\sqrt{s-\sin ^{2} \pi \tilde{y}}}-J_{s z}^{0} \\
& -\left.\frac{2}{\mu_{a}} B_{x}^{0}\right|_{x=0} \frac{\sin \pi \tilde{y}}{\sqrt{s-\sin ^{2} \pi \tilde{y}}} .
\end{array}\right\}
$$

We now refer back to the first of Equations (127), substitute Equation (168), and solve for $A_{2}$ to get

$$
A_{2}=-\frac{\mu_{a} \ln s}{4 \pi} K_{h}=-\left.\frac{\mu_{a} \ln s}{4 \pi}\left(H_{y}^{i}+H_{y+}^{0}-H_{y-}^{0}\right)\right|_{x=0} .
$$

So, finally, our solution for the current density is

$$
\left.\begin{array}{rl}
J_{s z}^{0}+j_{s z}^{0}(\tilde{y})= & K_{h} \frac{\cos \pi \tilde{y}}{\sqrt{s-\sin ^{2} \pi \tilde{y}}} \\
& -\left.\frac{2}{\mu_{a}} B_{x}^{0}\right|_{x=0} \frac{\sin \pi \tilde{y}}{\sqrt{s-\sin ^{2} \pi \tilde{y}}} .
\end{array}\right\}
$$

\section{C.3 The static problem for the electric field.}

Solution for the electric field problem proceeds along the same lines as for the magnetic field problem. We assume that $\bar{e}^{0}=-\nabla_{\tilde{r}} \Phi$. Then from the second of Equations (37) we have

$$
\nabla_{\tilde{r}}^{2} \Phi=0 .
$$

Suppose that $\Phi(\tilde{x}, \tilde{y})$ is a periodic function with period $p$. Then $\Phi(\tilde{x}, \tilde{y})$ can be expanded in a Fourier series as

$$
\Phi(\tilde{x}, \tilde{y})=\frac{c_{0}(\tilde{x})}{2}+\sum_{n=1}^{\infty} c_{n}(\tilde{x}) \cos 2 n \pi \tilde{y}+\sum_{n=1}^{\infty} d_{n}(\tilde{x}) \sin 2 n \pi \tilde{y}
$$

where 


$$
\left.\begin{array}{c}
c_{n}(\tilde{x})=2 \int_{-1 / 2}^{1 / 2} \Phi\left(\tilde{x}, \tilde{y}^{\prime}\right) \cos 2 n \pi \tilde{y}^{\prime} d \tilde{y}^{\prime}, n=0,1,2, \ldots \\
d_{n}(\tilde{x})=2 \int_{-1 / 2}^{1 / 2} \Phi\left(\tilde{x}, \tilde{y}^{\prime}\right) \sin 2 n \pi \tilde{y}^{\prime} d \tilde{y}^{\prime}, n=1,2, \ldots
\end{array}\right\}
$$

We now substitute this Fourier series representation into Equation (171). After comparing coefficients of the cosine and sine functions we have

$$
\left.\begin{array}{c}
\frac{\partial^{2} c_{0}(\tilde{x})}{\partial \tilde{x}^{2}}=0 \\
\frac{\partial^{2} c_{n}(\tilde{x})}{\partial \tilde{x}^{2}}-4 n^{2} \pi^{2} c_{n}(\tilde{x})=0, n=1,2, \ldots \\
\frac{\partial^{2} d_{n}(\tilde{x})}{\partial \tilde{x}^{2}}-4 n^{2} \pi^{2} d_{n}(\tilde{x})=0, n=1,2, \ldots
\end{array}\right\}
$$

The solutions for these equations are

$$
\left.\begin{array}{c}
c_{0}(\tilde{x})=\alpha \tilde{x}+\beta \\
c_{n+}(\tilde{x})=k_{1 n} e^{-2 n \pi \tilde{x}} \text { and } d_{n+}(\tilde{x})=l_{1 n} e^{-2 n \pi \tilde{x}}, \tilde{x}>0 \\
c_{n-}(\tilde{x})=k_{2 n} e^{+2 n \pi \tilde{x}} \text { and } d_{n-}(\tilde{x})=l_{2 n} e^{+2 n \pi \tilde{x}}, \tilde{x}>0
\end{array}\right\}
$$

where $\alpha, \beta, k$ and $l$ are constants.

We require that $\Phi(\tilde{x}, \tilde{y})$ go to zero as $\tilde{x} \rightarrow \pm \infty$ so that $c_{0}(\tilde{x})=0$. We then have

$$
\left.\begin{array}{l}
\Phi_{+}(\tilde{x}, \tilde{y})=\sum_{n=1}^{\infty} e^{-2 n \pi \tilde{x}}\left(k_{1 n} \cos 2 n \pi \tilde{y}+l_{1 n} \sin 2 n \pi \tilde{y}\right), \tilde{x}>0 \\
\Phi_{-}(\tilde{x}, \tilde{y})=\sum_{n=1}^{\infty} e^{+2 n \pi \tilde{x}}\left(k_{2 n} \cos 2 n \pi \tilde{y}+l_{2 n} \sin 2 n \pi \tilde{y}\right), \tilde{x}>0 .
\end{array}\right\}
$$

Since $\bar{e}^{0}=-\nabla_{\tilde{r}} \Phi$, we can write the electric fields as (noting that $e_{y}^{0}$ is continuous at $x=0$ so that $k_{1 n}=k_{2 n} \equiv k_{n}$ and $l_{1 n}=l_{2 n} \equiv l_{n}$ ) 


$$
\left.\begin{array}{c}
e_{x+}^{0}=\sum_{n=1}^{\infty} 2 n \pi e^{-2 n \pi \tilde{x}}\left(k_{n} \cos 2 n \pi \tilde{y}+l_{n} \sin 2 n \pi \tilde{y}\right), \tilde{x}>0 \\
e_{x-}^{0}=-\sum_{n=1}^{\infty} 2 n \pi e^{+2 n \pi \tilde{x}}\left(k_{n} \cos 2 n \pi \tilde{y}+l_{n} \sin 2 n \pi \tilde{y}\right), \tilde{x}<0 \\
e_{y+}^{0}=\sum_{n=1}^{\infty} 2 n \pi e^{-2 n \pi \tilde{x}}\left(k_{n} \sin 2 n \pi \tilde{y}-l_{n} \cos 2 n \pi \tilde{y}\right), \tilde{x}>0 \\
e_{y-}^{0}=\sum_{n=1}^{\infty} 2 n \pi e^{+2 n \pi \tilde{x}}\left(k_{n} \sin 2 n \pi \tilde{y}-l_{n} \cos 2 n \pi \tilde{y}\right), \tilde{x}>0 .
\end{array}\right\}
$$

From the fourth of Equations (37), we have

$$
\left.\left(\epsilon_{+} e_{x+}^{0}-\epsilon_{-} e_{x-}^{0}\right)\right|_{x=\tilde{x}=0}+\left.\left[\epsilon_{+}\left(E_{x+}^{0}+E_{x}^{i}\right)-\epsilon_{-} E_{x-}^{0}\right]\right|_{x=0}=P_{s}^{0}+\rho_{s}^{0} .
$$

The contribution from $\left.\left[\epsilon_{+}\left(E_{x+}^{0}+E_{x}^{i}\right)-\epsilon_{-} E_{x-}^{0}\right]\right|_{x=\tilde{x}=0}$ (call it $K_{e}$ ) is a function of $y$ and $z$ but is constant with respect to the fast variable so that we have

$$
K_{e}+\left.\left(\epsilon_{+} e_{x+}^{0}-\epsilon_{-} e_{x-}^{0}\right)\right|_{x=\tilde{x}=0}=P_{s}^{0}+\rho_{s}^{0} .
$$

If we substitute Equations (177) evaluated at $x=0$ into Equation (179) we get a Fourier series representation of the charge,

$$
\rho_{s}^{0}=K_{e}-P_{s}^{0}+\left(\epsilon_{+}+\epsilon_{-}\right) \sum_{n=1}^{\infty} 2 n \pi\left(k_{n} \cos 2 n \pi \tilde{y}+l_{n} \sin 2 n \pi \tilde{y}\right),
$$

whose coefficients are given by

$$
\left.\begin{array}{c}
K_{e}-P_{s}^{0}=\int_{-1 / 2}^{1 / 2} \rho_{s}^{0}\left(\tilde{y}^{\prime}\right) d \tilde{y}^{\prime} \\
\left(\epsilon_{+}+\epsilon_{-}\right) k_{n} 2 n \pi=2 \int_{-1 / 2}^{1 / 2} \rho_{s}^{0}\left(\tilde{y}^{\prime}\right) \cos 2 n \pi \tilde{y}^{\prime} d \tilde{y}^{\prime}, n=1,2, \ldots \\
\left(\epsilon_{+}+\epsilon_{-}\right) l_{n} 2 n \pi=2 \int_{-1 / 2}^{1 / 2} \rho_{s}^{0}\left(\tilde{y}^{\prime}\right) \sin 2 n \pi \tilde{y}^{\prime} d \tilde{y}^{\prime}, n=1,2, \ldots
\end{array}\right\}
$$

Now, in the gap, we have that $P_{s}^{0}+\rho_{s}^{0}=0$, or $\rho_{s}^{0}=-P_{s}^{0}$ so we can split the integrals of Equations (181) into those in the gap and those on the strip. After a little algebra we have 


$$
\left.\begin{array}{c}
K_{e}-P_{s}^{0} \frac{b}{p}=\int_{-b / 2 p}^{b / 2 p} \rho_{s}^{0}\left(\tilde{y}^{\prime}\right) d \tilde{y}^{\prime} \\
k_{n}=\frac{1}{2 \epsilon_{a} n \pi} \int_{-b / 2 p}^{b / 2 p}\left[P_{s}^{0}+\rho_{s}^{0}\left(\tilde{y}^{\prime}\right)\right] \cos 2 n \pi \tilde{y}^{\prime} d \tilde{y}^{\prime} \\
l_{n}=\frac{1}{2 \epsilon_{a} n \pi} \int_{-b / 2 p}^{b / 2 p} \rho_{s}^{0}\left(\tilde{y}^{\prime}\right) \sin 2 n \pi \tilde{y}^{\prime} d \tilde{y}^{\prime}
\end{array}\right\}
$$

where

$$
\epsilon_{a}=\frac{\epsilon_{+}+\epsilon_{-}}{2}
$$

On the strip, $e_{y}^{0}+\left.E_{y}^{0}\right|_{x=0}=0$ so that $e_{y}^{0}=-\left.E_{y}^{0}\right|_{x=0} \cdot{ }^{5}$ From the relation between $e_{y}^{0}$ and $\Phi$ we then have on the strip

$$
\frac{\partial \Phi}{\partial \tilde{y}}=\left.E_{y}^{0}\right|_{x=0}
$$

so that

$$
\Phi=\left.E_{y}^{0}\right|_{x=0} \tilde{y}+\Phi_{0}
$$

where $\Phi_{0}$ is a constant of integration. We now substitute Equation (185) along with the second and third of Equations (182) into Equation (176) evaluated at $\tilde{x}=0$. We then have

$$
\begin{aligned}
2 \epsilon_{a} \pi\left[\left.E_{y}^{0}\right|_{x=0} \tilde{y}+\Phi_{0}\right] & =\sum_{n=1}^{\infty} \frac{1}{n} \cos 2 n \pi \tilde{y} \int_{-b / 2 p}^{b / 2 p}\left[P_{s}^{0}+\rho_{s}^{0}\left(\tilde{y}^{\prime}\right)\right] \cos 2 n \pi \tilde{y}^{\prime} d \tilde{y}^{\prime} \\
& +\sum_{n=1}^{\infty} \frac{1}{n} \sin 2 n \pi \tilde{y} \int_{-b / 2 p}^{b / 2 p} \rho_{s}^{0}\left(\tilde{y}^{\prime}\right) \sin 2 n \pi \tilde{y}^{\prime} d \tilde{y}^{\prime}
\end{aligned}
$$

Interchanging summation and integration gives

\footnotetext{
${ }^{5}$ Here, the total zeroth order electric field includes the incident field.
} 


$$
\left.\begin{array}{rl}
2 \epsilon_{a} \pi\left[\left.E_{y}^{0}\right|_{x=0} \tilde{y}+\Phi_{0}\right] & =\int_{-b / 2 p}^{b / 2 p}\left[P_{s}^{0}+\rho_{s}^{0}\left(\tilde{y}^{\prime}\right)\right] \sum_{n=1}^{\infty} \frac{1}{n} \cos 2 n \pi \tilde{y} \cos 2 n \pi \tilde{y}^{\prime} d \tilde{y}^{\prime} \\
& +\int_{-b / 2 p}^{b / 2 p} \rho_{s}^{0}\left(\tilde{y}^{\prime}\right) \sum_{n=1}^{\infty} \frac{1}{n} \sin 2 n \pi \tilde{y} \sin 2 n \pi \tilde{y}^{\prime} d \tilde{y}^{\prime}
\end{array}\right\}
$$

We now have an integral equation for $\rho_{s}^{0}(\tilde{y})$ which is of the same form as Equation (134). To find the solutions to Equation (187) we merely make the following replacements in Equations (168).

$$
\left.\begin{array}{c}
-\left.\left.\frac{1}{\mu_{a}} B_{x}^{0}\right|_{x=0} \rightarrow \epsilon_{a} E_{y}^{0}\right|_{x=0} \\
\frac{1}{\mu_{a}} A_{2} \rightarrow \epsilon_{a} \Phi_{0} . \\
J_{s z}^{0} \rightarrow P_{s}^{0}
\end{array}\right\}
$$

This gives

$$
\left.\begin{array}{rl}
P_{s}^{0}+\rho_{s}^{0}(\tilde{y})= & -4 \pi \epsilon_{a} \frac{\Phi_{0}}{\ln s} \frac{\cos \pi \tilde{y}}{\sqrt{s-\sin ^{2} \pi \tilde{y}}} \\
& +\left.2 \epsilon_{a} E_{y}^{0}\right|_{x=0} \frac{\sin \pi \tilde{y}}{\sqrt{s-\sin ^{2} \pi \tilde{y}}}
\end{array}\right\}
$$

We proceed with similar arguments as in the previous section to evaluate the constant of Equation (189) to get

$$
\Phi_{0}=-\frac{\ln s}{4 \pi \epsilon_{a}} K_{e}=-\left.\frac{\ln s}{4 \pi \epsilon_{a}}\left[\epsilon_{+}\left(E_{x}^{i}+E_{x+}^{0}\right)-\epsilon_{-} E_{x-}^{0}\right]\right|_{x=0} .
$$

Our solution for the charge is then 


$$
\left.\begin{array}{rl}
P_{s}^{0}+\rho_{s}^{0}(\tilde{y})= & K_{e} \frac{\cos \pi \tilde{y}}{\sqrt{s-\sin ^{2} \pi \tilde{y}}} \\
& +\left.2 \epsilon_{a} E_{y}^{0}\right|_{x=0} \frac{\sin \pi \tilde{y}}{\sqrt{s-\sin ^{2} \pi \tilde{y}}}
\end{array}\right\}
$$

\section{C.4 Summary of the static solutions.}

The following is a summary of the relevant equations of the static fields.

$$
\begin{gathered}
h_{x \pm}^{0}=-\frac{1}{\mu_{ \pm}} \sum_{n=1}^{\infty} 2 n \pi e^{\mp 2 n \pi \tilde{x}}\left(g_{n} \sin 2 n \pi \tilde{y}-f_{n} \cos 2 n \pi \tilde{y}\right) \\
h_{y \pm}^{0}= \pm \frac{1}{\mu_{ \pm}} \sum_{n=1}^{\infty} 2 n \pi e^{\mp 2 n \pi \tilde{x}}\left(g_{n} \cos 2 n \pi \tilde{y}+f_{n} \sin 2 n \pi \tilde{y}\right) \\
2 n \pi g_{n}=\mu_{a} \int_{-b / 2 p}^{b / 2 p}\left[J_{s z}^{0}+j_{s z}^{0}\left(\tilde{y}^{\prime}\right)\right] \cos 2 n \pi \tilde{y}^{\prime} d \tilde{y}^{\prime} \\
2 n \pi f_{n}=\mu_{a} \int_{-b / 2 p}^{b / 2 p} j_{s z}^{0}\left(\tilde{y}^{\prime}\right) \sin 2 n \pi \tilde{y}^{\prime} d \tilde{y}^{\prime} \\
\mu_{a}=\frac{2 \mu_{+} \mu_{-}}{\mu_{+}+\mu_{-}}
\end{gathered}
$$

where

$$
\left.\begin{array}{rl}
J_{s z}^{0}+j_{s z}^{0}(\tilde{y})= & K_{h} \frac{\cos \pi \tilde{y}}{\sqrt{s-\sin ^{2} \pi \tilde{y}}} \\
& -\left.\frac{2}{\mu_{a}} B_{x}^{0}\right|_{x=0} \frac{\sin \pi \tilde{y}}{\sqrt{s-\sin ^{2} \pi \tilde{y}}} \\
J_{s z}^{0}=K_{h}= & \left.\left(H_{y}^{i}+H_{y+}^{0}-H_{y-}^{0}\right)\right|_{x=0}
\end{array}\right\}
$$

and 


$$
\begin{gathered}
e_{x \pm}^{0}= \pm \sum_{n=1}^{\infty} 2 n \pi e^{\mp 2 n \pi \tilde{x}}\left(k_{n} \cos 2 n \pi \tilde{y}+l_{n} \sin 2 n \pi \tilde{y}\right) \\
e_{y \pm}^{0}=\sum_{n=1}^{\infty} 2 n \pi e^{\mp 2 n \pi \tilde{x}}\left(k_{n} \sin 2 n \pi \tilde{y}-l_{n} \cos 2 n \pi \tilde{y}\right) \\
2 n \pi k_{n}=\frac{1}{\epsilon_{a}} \int_{-b / 2 p}^{b / 2 p}\left[P_{s}^{0}+\rho_{s}^{0}\left(\tilde{y}^{\prime}\right)\right] \cos 2 n \pi \tilde{y}^{\prime} d \tilde{y}^{\prime} \\
2 n \pi l_{n}=\frac{1}{\epsilon_{a}} \int_{-b / 2 p}^{b / 2 p} \rho_{s}^{0}\left(\tilde{y}^{\prime}\right) \sin 2 n \pi \tilde{y}^{\prime} d \tilde{y}^{\prime} \\
\epsilon_{a}=\frac{\epsilon_{+}+\epsilon_{-}}{2}
\end{gathered}
$$

where

$$
\left.\begin{array}{rl}
P_{s}^{0}+\rho_{s}^{0}(\tilde{y})= & K_{e} \frac{\cos \pi \tilde{y}}{\sqrt{s-\sin ^{2} \pi \tilde{y}}} \\
& +\left.2 \epsilon_{a} E_{y}^{0}\right|_{x=0} \frac{\sin \pi \tilde{y}}{\sqrt{s-\sin ^{2} \pi \tilde{y}}} \\
P_{s}^{0}=K_{e}= & {\left.\left[\epsilon_{+}\left(E_{x}^{i}+E_{x+}^{0}\right)-E_{x-}^{0}\right]\right|_{x=0}}
\end{array}\right\}
$$

and

$$
\begin{aligned}
& s=\frac{1}{2}\left(1-\cos \frac{\pi b}{p}\right)=\sin ^{2} \frac{\pi b}{2 p} \\
& c=\frac{1}{2}\left(1+\cos \frac{\pi b}{p}\right)=\cos ^{2} \frac{\pi b}{2 p} .
\end{aligned}
$$

We can now see that it is easy to combine Equations (193) and the second pair of Equations (192). This is facilitated by the knowledge of the even and odd parts of the current function. The result is

$$
\left.\begin{array}{l}
2 n \pi g_{n}=2 \mu_{a} K_{h} \int_{0}^{b / 2 p} \frac{\cos \pi \tilde{y}^{\prime}}{\sqrt{s-\sin ^{2} \pi \tilde{y}^{\prime}}} \cos 2 n \pi \tilde{y}^{\prime} d \tilde{y}^{\prime} \\
2 n \pi f_{n}=-\left.4 B_{x}^{0}\right|_{x=0} \int_{0}^{b / 2 p} \frac{\sin \pi \tilde{y}^{\prime}}{\sqrt{s-\sin ^{2} \pi \tilde{y}^{\prime}}} \sin 2 n \pi \tilde{y}^{\prime} d \tilde{y}^{\prime} .
\end{array}\right\}
$$


Similar algebraic manipulations can be used to combine Equations (195) and the second pair of Equations (194). We get

$$
\left.\begin{array}{rl}
2 n \pi k_{n} & =\frac{2}{\epsilon_{a}} K_{e} \int_{0}^{b / 2 p} \frac{\cos \pi \tilde{y}^{\prime}}{\sqrt{s-\sin ^{2} \pi \tilde{y}^{\prime}}} \cos 2 n \pi \tilde{y}^{\prime} d \tilde{y}^{\prime} \\
2 n \pi l_{n} & =\left.4 E_{y}^{0}\right|_{x=0} \int_{0}^{b / 2 p} \frac{\sin \pi \tilde{y}^{\prime}}{\sqrt{s-\sin ^{2} \pi \tilde{y}^{\prime}}} \sin 2 n \pi \tilde{y}^{\prime} d \tilde{y}^{\prime} .
\end{array}\right\}
$$

We now define

$$
\left.\begin{array}{c}
\vartheta_{n}=2 \int_{0}^{b / 2 p} \frac{\cos \pi \tilde{y}^{\prime}}{\sqrt{s-\sin ^{2} \pi \tilde{y}^{\prime}}} \cos 2 n \pi \tilde{y}^{\prime} d \tilde{y}^{\prime} \\
\varphi_{n}=-4 \int_{0}^{b / 2 p} \frac{\sin \pi \tilde{y}^{\prime}}{\sqrt{s-\sin ^{2} \pi \tilde{y}^{\prime}}} \sin 2 n \pi \tilde{y}^{\prime} d \tilde{y}^{\prime}
\end{array}\right\}
$$

so that we can write complete expressions for the fields. They are

$$
\begin{aligned}
& h_{x \pm}^{0}=-\frac{1}{\mu_{ \pm}} \sum_{n=1}^{\infty} e^{\mp 2 n \pi \tilde{x}}\left[\mu_{a} K_{h} \vartheta_{n} \sin 2 n \pi \tilde{y}-\left.B_{x}^{0}\right|_{x=0} \varphi_{n} \cos 2 n \pi \tilde{y}\right] \\
& h_{y \pm}^{0}= \pm \frac{1}{\mu_{ \pm}} \sum_{n=1}^{\infty} e^{\mp 2 n \pi \tilde{x}}\left[\mu_{a} K_{h} \vartheta_{n} \cos 2 n \pi \tilde{y}+\left.B_{x}^{0}\right|_{x=0} \varphi_{n} \sin 2 n \pi \tilde{y}\right] \\
& e_{x \pm}^{0}= \pm \sum_{n=1}^{\infty} e^{\mp 2 n \pi \tilde{x}}\left[\frac{K_{e}}{\epsilon_{a}} \vartheta_{n} \cos 2 n \pi \tilde{y}-\left.E_{y}^{0}\right|_{x=0} \varphi_{n} \sin 2 n \pi \tilde{y}\right] \\
& e_{y \pm}^{0}=\sum_{n=1}^{\infty} e^{\mp 2 n \pi \tilde{x}}\left[\frac{K_{e}}{\epsilon_{a}} \vartheta_{n} \sin 2 n \pi \tilde{y}+\left.E_{y}^{0}\right|_{x=0} \varphi_{n} \cos 2 n \pi \tilde{y}\right] .
\end{aligned}
$$




\section{References}

[1] Horace Lamb, "On the reflection and transmission of electric waves by a metallic grating," Proc. London Math. Soc., ser. 1, vol. 29, pp. 523-544, 1898.

[2] Robin I. Primich, "Some electromagnetic transmission and reflection properties of strip grating" IRE Trans. Antennas and Propagation, pp. 176-182, 1957.

[3] H. A. Kalhor and Armand, "Scattering of waves by gratings of conducting cylinders," Proc. IEE., vol. 122, no. 3, pp. 245-248, 1975.

[4] H. A. Kalhor, "Diffraction of electromagnetic waves by plane metallic gratings," J. Opt. Soc. Am., vol. 68., no 9, pp. 1202-1205, 1978.

[5] J. Y. Suratteau, M. Cadilhac, and R. Petit, "The perfectly conducting wire grating; Computation of the diffracted field from Maxwell's equations and Hamilton's cononical system," IEEE Trans. Ant. Prop., vol. AP-33, no. 4, pp. 404-408, 1985.

[6] I. Palocz and A. A. Oliner, "Equivalent network of a multimode planar grating," IEEE Trans. Micr. Theory Tech., vol. MTT-18, no. 5, pp. 244-252, 1970.

[7] M. Guglielmi and A. A. Oliner, "Multimode network description of a planar periodic metal-strip grating at a dielectric interface-Part I: Rigorous network formulation," IEEE Trans. Micr. Theory Tech., vol. MTT-37, no. 3, pp. 534-541, 1989.

[8] M. Guglielmi and A. A. Oliner, "Multimode network description of a planar periodic metal-strip grating at a dielectric interface-Part II: Small-aperture and small-obstacle solutions," IEEE Trans. Micr. Theory Tech., vol. MTT-37, no. 3, pp. 542-551, 1989.

[9] M. Guglielmi and H. Hochstadt, "Multimode network description of a planar periodic metal-strip grating at a dielectric interface-Part III: Rigorous solution," IEEE Trans. Micr. Theory Tech., vol. MTT-37, no. 5, pp. 902-909, 1989. 
[10] Tokio Sakurai, "Electromagnetic phenomena on ribbon elements grating," J. Phys. Soc. Japan, vol. 5, pp. 203-207, 1950.

[11] A. N. Sivov, "Electrodynamic theory of a dense plane grating of parallel conductors," Radio Eng. Electron. Phys., vol. 6, pp. 429-440, 1961.

[12] M. I. Kontorovich, "Averaged boundary conditions at the surface of a grating with square mesh," Radio Engineering, vol. 17, pp. 1446-1454, 1962 .

[13] L. A. Vainshtein (Wainstein), "On the electrodynamic theory of grids," in High-Power Electronics. Oxford: Pergamon Press, pp. 14-48, 1966.

[14] A. I. Adonina and V. V. Shcherbak, "Diffraction of electromagnetic waves obliquely incident on a plane metallic grating with a screen and a magnetodielectric," Soviet Phys.-Tech. Phys., vol. 9, pp. 133-137, 1964.

[15] A. I. Adonina, and V. V. Shcherbak, "Equivalent boundary conditions at a metal grating situated between two magnetic materials," Soviet Phys.-Tech. Phys., vol. 9, pp. 261-263, 1964.

[16] M. I. Astrakhan, "Reflecting and screening properties of plane wire grids," Radio Engineering, vol. 23, no. 1, pp. 76-83, 1968.

[17] L. Lewin, Theory of Waveguides, London, Newnes-Butterworths, 1975.

[18] J. L. Lions, Some Methods in the Mathematical Analysis of Systems and Their Control, New York, Gordon and Breach, Science Publishers, Inc., 1981.

[19] M. Codegone, "On the acoustic impedance condition for ondulated boundary," Ann. Inst. Henri Poincaré Sect. A: Phys. Théorique, vol. 36, pp. 1-18, 1982.

[20] G. Nguetseng, "Problèmes d'écrans perforés pour l'èquation de Laplace," $M^{2}$ AN Modelis. Math. Anal. Numér., vol. 19, pp. 33-63, 1985. 
[21] E. Sanchez-Palencia, Non-Homogeneous Media and Vibration Theory (Lecture Notes in Physics no. 127). Berlin: Springer-Verlag, 1980, pp. 68-77.

[22] A. Bensoussan, J. L. Lions, G. Papunicolaou, Asymptotic Analysis for Periodic Structures, North Holand, Amsterdam, 1978.

[23] I. S. Gradshteyn and I. M. Ryzhik, Table of Integrals, Series, and Products, New York, Academic Press, Inc., 1980.

[24] S. Ye. Bankov and I. V. Levchenko, "Equivalent boundary conditions for a closely spaced ribbon grating at the interface of two media," Soviet Jn. Comm. Tech. and Electron., vol. 34, no. 5, pp. 67-72, 1989.

[25] J. Schwinger and D.S. Saxon, Discontinuities in Waveguides, New York, Gordon and Breach Science Publishers, 1968. 